\title{
Targeted chromosomal Escherichia coli:dnaB exterior surface residues regulate DNA helicase behavior to maintain genomic stability and organismal fitness
}

\author{
Megan S. Behrmann, Himasha M. Perera, Joy M. Hoang, Trisha A. Venkat, and Michael A. Trakselis* \\ Department of Chemistry and Biochemistry, Baylor University, Waco, Texas, 76798, USA \\ *To whom correspondence should be addressed: *Michael A. Trakselis, One Bear Place \#97348, Waco, TX 76798-7348. Tel 254- \\ 710-2581; Email: michael_trakselis@,baylor.edu
}

\begin{abstract}
$\underline{\text { Abstract }}$
Helicase regulation is vital for replisome progression, where the helicase enzyme functions to unwind duplex DNA and aids in the coordination of replication fork activities. Currently, mechanisms for helicase regulation that involve interactions with both DNA strands through a steric exclusion and wrapping (SEW) model and conformational shifts between dilated and constricted states have been examined in vitro. To better understand the mechanism and cellular impact of helicase regulation, we used CRISPRCas9 genome editing to study four previously identified SEW-deficient mutants of the bacterial replicative helicase DnaB. We discovered that these four SEW mutations stabilize constricted states, with more fully constricted mutants having a generally greater impact on genomic stress, suggesting a dynamic model for helicase regulation that involves both excluded strand interactions and conformational states. These $d n a B$ mutations result in increased DNA damage and chromosome complexity, less stable genomes, and ultimately less viable and fit strains. Notably, while two mutations stabilized fully constricted states, they have distinct effects on genomic stability, suggesting a complex relationship between helicase regulation mechanisms and faithful, efficient DNA replication. This work explores the genomic impacts of helicase dysregulation in vivo, supporting a combined dynamic regulatory mechanism involving SEW and conformational changes and relates current mechanistic understanding to functional helicase behavior.
\end{abstract}

Keywords: DNA replication; helicase; replisome; coupling; DnaB; unwinding

\section{Introduction}

Faithful and efficient DNA replication is a fundamental life process that is the result of complex interactions between a diverse collection of enzymes. Proximal to this process is the DNA helicase enzyme, a hexameric protein ensemble that separates double-stranded DNA (dsDNA) for synthesis and coordinates replicative actions. The functional mechanism for unwinding of this toroidal-shaped enzyme is well studied, however the method by which the helicase is regulated remains unresolved $[1,2]$. It is known that the helicase unwinds by steric exclusion (SE); a mechanism that involves encircling and translocating on one strand with a particular polarity, while the complementary strand is excluded from the central channel $[2,3]$. The excluded strand has also been implicated in helicase regulation [4-9] by interacting electrostatically on the exterior of many different helicases from multiple organisms, establishing the steric exclusion and wrapping (SEW) model for unwinding [10-13]. Other mechanisms for helicase regulation have been proposed [14-19], but little has been done to examine the cellular consequences of helicase mechanism dysregulation in vivo.

Escherichia coli (E. coli) DnaB is a homohexameric superfamily 4 (SF4) helicase that encircles and translocates along the lagging strand in the 5'-3' direction [20, 21]. Not only are SF4 helicases well characterized, but $E$. coli is a well-tested model organism, making this an ideal system for investigating replisome mechanics in vivo. The SE model provides a mechanism for single-strand DNA (ssDNA) translocation and prevents immediate reannealing of the DNA strands during unwinding; while the SEW model adds a dynamic interaction with the excluded strand to modulate enzyme activity. Biochemical analyses of DnaB indicated that a stable interaction with the excluded strand restricts unwinding, likely acting as a brake to slow helicase progression [4]. Site-specific external SEW mutations of DnaB resulted in 20 to 50fold increases in DNA unwinding in vitro. This regulation may be important in vivo to limit separation of DnaB from the replisome, which may occur during Okazaki fragment priming or during helicasepolymerase decoupling [22, 23]. Functionally, this would promote coordinated helicase-polymerase coupled DNA replication and aid in preventing ssDNA buildup from uncontrolled unwinding to limit chromosomal breaks [24].

In addition to direct interactions with DNA strands, the DnaB hexamer also undergoes a large conformational change upon interaction with specific replisome components: clamp-loader complex (CLC) (specifically tau), primase (DnaG), and the helicase loader (DnaC). At least two distinct conformations of the DnaB helicase have been observed: a dilated state that favors interaction with DnaG [25] and was shown to unwind similar to wild-type (WT) DnaB in vitro [14] and a constricted state that favors interaction with DnaC [26] and resulted in rapid unwinding relative to WT that was further stimulated by addition of tau [14]. These conformational dynamics likely also affect the binding affinity of the excluded strand, ultimately controlling the unwinding speed for the replisome.

In this report, we confirm that the faster unwinding SEW DnaB mutants also stabilize a constricted helicase conformation, indicating that both excluded strand access to the exterior surface and the conformational state of the helicase contribute to the structure/function mechanism for regulating unwinding speed. Identical site-specific genomic mutations of $E$. coli $d n a B$ were engineered using CRISPR-Cas9 
Behrmann et al., 25/05/2021 - preprint copy - BioRxiv

editing to investigate the effects of helicase regulation on cellular fitness and overall genomic stability. Generally, dnaB mutants grew slower, were outcompeted by the parental strain, and displayed a filamentous cell phenotype, indicating more genomic and cellular stress. Fluorescence activated cell sorting (FACS) and quantitative PCR (qPCR) analysis indicated higher chromosome numbers and increased ori:ter ratio indicating dysregulation of replication initiation that may be caused by altered DnaB loading for initiation for some of the mutants. A subsection of $d n a B$ mutants had significant genome instability, demonstrated by upregulation of the SOS response, increased mutagenesis, and prevalent double strand breaks. This work has important implications regarding the impact and importance of regulation of replisome speed on genomic stability, replication efficiency, and cellular fitness.

\section{$\underline{\text { Results }}$}

DnaB SEW mutants favor a constricted conformation. The DnaB hexamer is known to adopt both constricted and dilated conformations. DnaC stabilizes a constricted cracked conformation (lock washer) for loading [26]; the dilated conformation favors DnaG recruitment for priming [25]; and the $\tau$ subunit of the CLC stimulates the closed constricted state to couple rapid DNA synthesis with unwinding [14]. The constricted conformation is more efficient for translocation and unwinding but is unable to transverse over duplex DNA. To determine whether our DnaB SEW protein mutants enforce one confirmation over the other to explain the increased DNA unwinding rates [4], we utilized a duplexed fluorescence translocation fork assay, similar to that described previously (Fig. 1) [14].

Purified WT and mutant DnaB enzymes were incubated with a forked Cy3-fluorescently labeled and black hole quencher (BHQ) substrate, containing a duplex region prior to a 3' fork displaced strand (Fig. 1B \& Fig. S1). DnaB is unable to unwind a 5' single arm substrate [27], and so, it must translocate over the duplex region to separate the Cy3-reporter strand. Only DnaB hexamers that can adopt a dilated state or fluctuate between conformational states can move over the duplex to unwind the Cy3-reporter strand from the BHQ strand, resulting in an increase in the fluorescent signal upon addition of ATP (Fig. 1B) [14]. WT DnaB is able to freely switch between conformations and unwound $43 \%$ of the total DNA substrate (Fig. 1A). Both DnaB (K180A) and DnaB (R328/9A) have essentially no increases in fluorescence over time, unwinding only $\sim 5 \%$, similar to the no ATP negative control. In a previous report, both $\mathrm{K} 180 \mathrm{~A}$ and $\mathrm{R} 328 / 9 \mathrm{~A}$ had 20 -fold increases in unwinding rates on traditional forked substrates [4]; however, in this duplex translocation assay, both K180A and R328/9A mutants are unable to translocate over duplex DNA and therefore maintain a static fully constricted conformation. DnaB(R74A) and DnaB(R164A) both show moderate increases in fluorescence with $18 \%$ and $15 \%$ Cy3 strand unwound, respectively. This demonstrates that although R74A and R164A can switch to a dilated state, these mutations shift the equilibria towards the constricted state, consistent with their 3-6-fold faster unwinding of traditional forks $[4,14]$ but can still fluctuate somewhat to a dilated conformation. These mutants, (R74A and R164A), present states more moderately constricted and intermediate than previously seen.

In vivo dnaB mutations limit growth and generate stress. To determine whether faster in vitro DNA unwinding with preferential constricted conformations for DnaB (R74A, R164A, K180A, and R328/9A) have detrimental effects on replication speed, genomic stability, and organismal fitness, we created site-specific genomic dnaB point mutations using CRISPR-Cas9 editing (Fig. S1). The exponential growth rate for all $d_{n a B}$ mutants was monitored at $O D_{600}$ in rich media and compared to the parental strain using a plate reader maintained at 32 ${ }^{\circ} \mathrm{C}$. The maximal exponential growth rate for most of the $d n a B$ mutants was significantly decreased compared to the parental strain with the exception of $d n a B: R 74 A$ (Fig. 2A). Interestingly, within the mutant $d n a B$ strains, there is variability in the fitted exponential growth curves. $d n a B: W T$ and $d n a B: R 74 A$ increase in density during log phase at a rate of approximately $0.18 \pm 0.01$ per hour. $d n a B: K 180 A$ has a growth rate of $0.09 \pm 0.00$, half that of $d n a B: W T$ and the slowest of all the mutants (Fig. 2B). dnaB:R164A increases at a rate of $0.13 \pm 0.01$ per hour, nearly 1.5 times slower than the parental strain. dnaB:R328/9A has a growth rate of $0.15 \pm 0.02$ per hour, similar but still significantly slower than $d n a B: W T$. A reduction in overall growth rate may indicate that these helicase
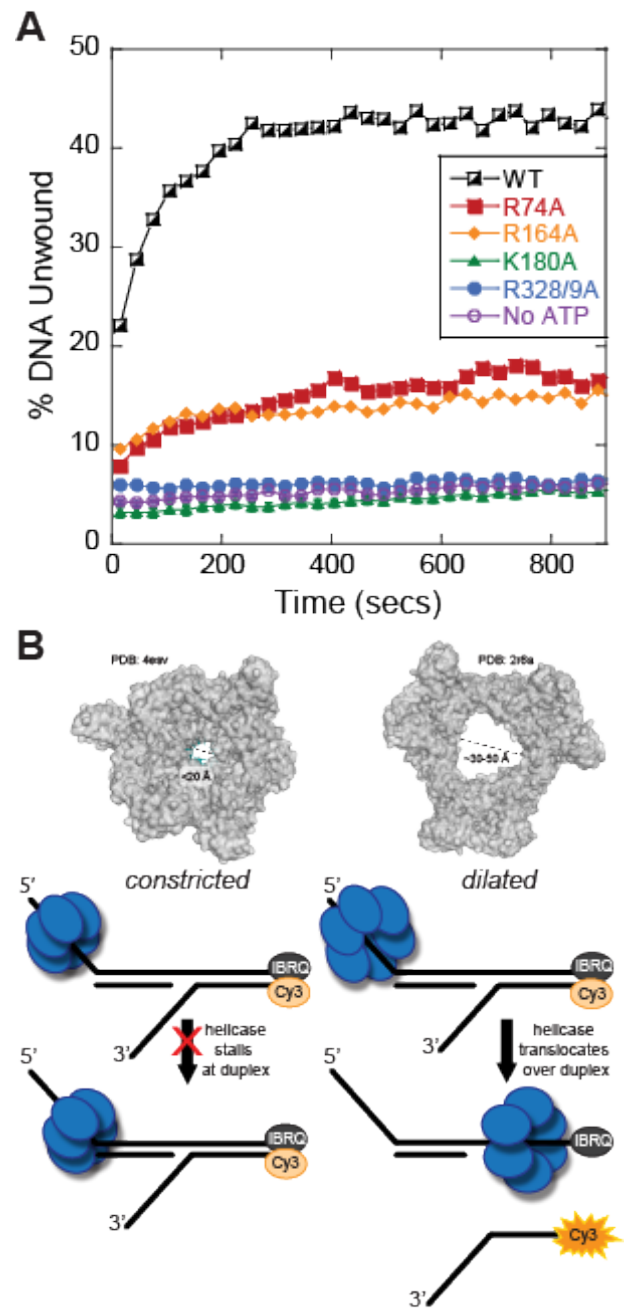

Fig. 1: DnaB mutants induce a constricted state measured in a fluorescence duplex translocation assay. (A) A plot comparing the amount of Cy3-DNA unwound for each mutant (colored consistently throughout) as a function of time. Average of $n=7$ replicates shown. Data is fit to Equation 1. Error bars represent \pm standard deviation (SD) and are within symbols where not visible. Negative control is WT DnaB with all the reaction components excluding ATP (No ATP, purple). Rates of unwinding are $10.7 \pm 0.5 \times 10^{-3} \mathrm{~s}^{-1}$ for WT (black squares), 4.0 $\pm 0.4 \times 10^{-3} \mathrm{~s}^{-1}$ for R74A (red squares), $4.5 \pm 0.9 \times 10^{-3} \mathrm{~s}^{-1}$ for R164A (orange diamonds), $0.0 \pm 0.3 \times 10^{-3} \mathrm{~s}^{-1}$ for K180A (green triangles), $0.0 \pm 1.3 \times 10^{-3} \mathrm{~s}^{-1}$ for R328/9A (blue dots), and $0.0 \pm 0.7 \times 10^{-3} \mathrm{~s}^{-1}$ for no ATP control (purple circles). (B) Crystal structures of a constricted DnaB helicase bound to ssDNA $(G$. stearothermophilus, PDBD: 4esv), corresponding dilated GstDnaB helicase (PDBID: 2r6a), and a schematic of the substrate design for duplex translocation assay liberating the $\mathrm{Cy} 3$ strand for increased fluorescence. 
A

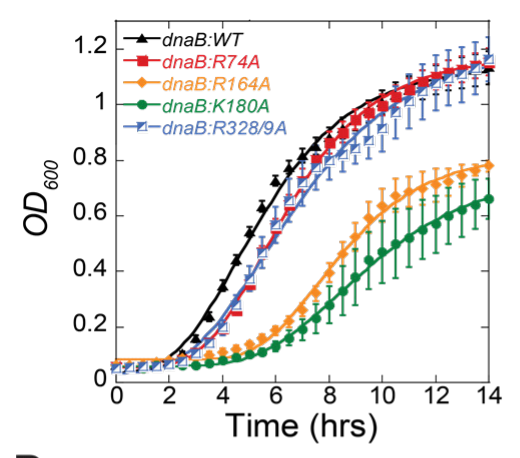

B

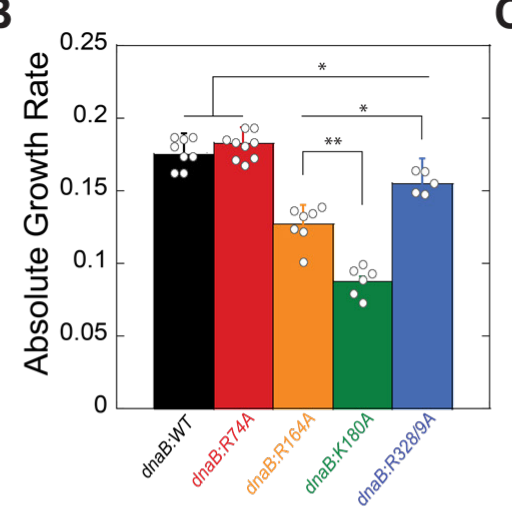

C

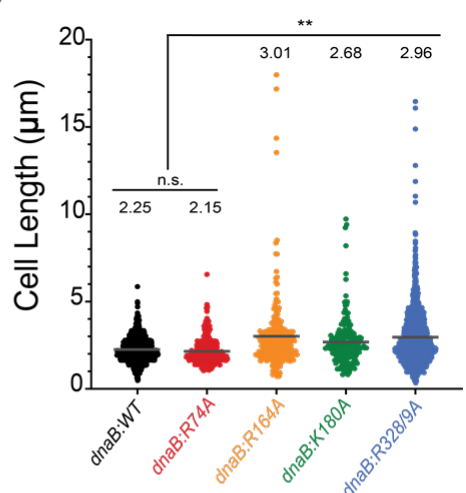

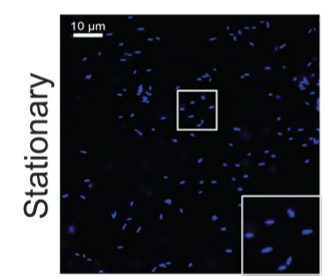

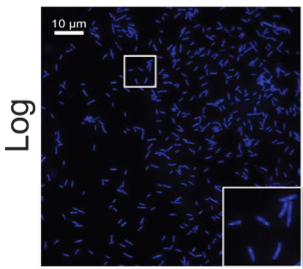

dnaB:WT
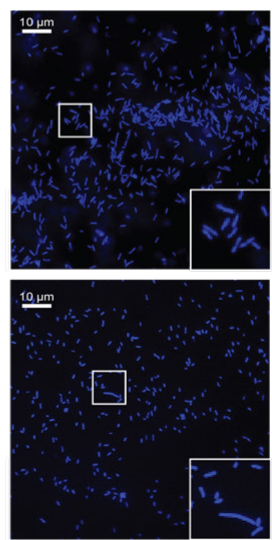

dnaB:R74A
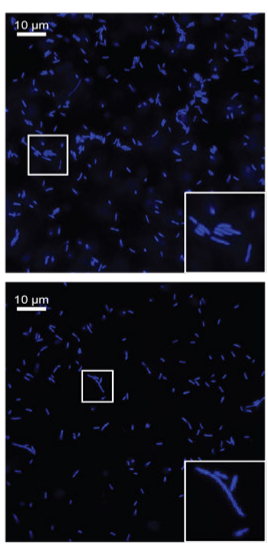

dnaB:R164A

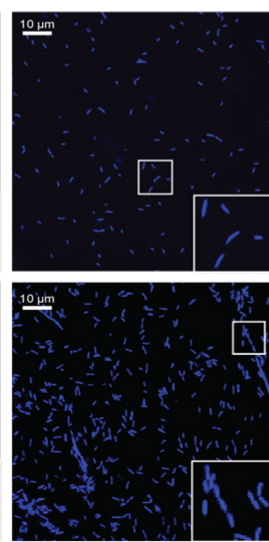

dnaB:K180A

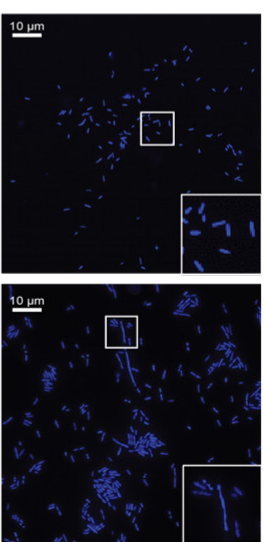

dnaB:R328/9A

Fig. 2: Growth and visualization of cell morphology for $\boldsymbol{d n a B : m u t}$ and $\boldsymbol{W T}$ strains (A) Growth curves of indicated $E$. coli strains grown at $32^{\circ} \mathrm{C}$ are fitted to Equation 2. Data plotted is the mean for three trials of three technical replicates each $(n=9)$, and error bars represent $\pm \mathrm{SD}(\mathbf{B})$ The averaged exponential growth rate for each strain is plotted for comparison. Individual data is presented with open circles. Black bars indicate statistically significant differences, where $\mathrm{p}$-values are $*<0.05$ and $* *<0.01$. (C) The cell lengths were measured by blinded visual quantification for $\mathrm{n} \geq 200$ cells, and the average cell length is reported above the data points (grey bar) for each sample. Statistically significant differences are indicated, where p-values are $* *<0.01$. n.s. is not significant. (D) Stationary and log phase cells are stained with DAPI and imaged using epifluorescence microscopy. Images shown are representative of the population observed. Magnified inlays emphasize areas of interest.

mutations are causing genomic or cellular stress, which in bacteria is often typified by cellular elongation. $0.15 \pm 0.02$ per hour, similar but still significantly slower than $d n a B: W T$. A reduction in overall growth rate may indicate that these helicase mutations are causing genomic or cellular stress, which in bacteria is often typified by cellular elongation. To investigate this, we exposed log phase samples to DAPI, imaged using an epifluorescent light microscope, measured the cell length, and analyzed these strains blindly (Fig. 2C). All mutants, except $d n a B: R 74 A$, had significantly elongated cells in $\log$ phase relative to $d n a B: W T$, which had an average cell size of $2.25 \pm 0.02 \mu \mathrm{m}$ with sizes ranging up to 5 and $6 \mu \mathrm{m}$. dnaB:R164A and $d n a B: R 328 / 9 A$ strains averaged $3.01 \pm 0.14$ and $2.96 \pm 0.14 \mu \mathrm{m}$ per cell, with $\operatorname{dnaB}: R 328 / 9 A$ having the largest population of longer cells $(>5 \mu \mathrm{m})$ and cells ranging to over $15 \mu \mathrm{m}$ in length. dnaB:R164A had the longest cells reaching $20 \mu \mathrm{m}$ in length. $d n a B: K 180 A$ had an average cell length of $2.68 \pm 0.10 \mu \mathrm{m}$ with a maximum recorded size of $10 \mu \mathrm{m}$. Representative images of cell populations show visual increases in the filamented cell population, with inlays highlighting representative groups (Fig. 2D). Additionally, Large aggregates of filamented cells were observed for $d n a B: K 180 A$ and dnaB:R328/9A. Actively dividing cells can clearly be seen in all the log phase cultures, including the parental strain, and care was taken not to confuse them with filamenting cells.

We also examined stationary phase samples of the mutants (Fig. S2), and found that most strains maintained an average of about $2 \mu \mathrm{m}$ in cell length with a maximum length of $5.1 \mu \mathrm{m}$, with the exception of
dnaB:R164A. The dnaB:R164A mutant strain had had an average cell length of $2.6 \pm 0.9 \mu \mathrm{m}$ and a maximum cell length of $10.6 \mu \mathrm{m}$ and was significantly different than WT.

SEW is important for strain efficacy and survival. To understand how the differences in growth rate and cell morphology affected overall strain fitness, we utilized a colorimetric direct growth competition assay [28-31]. The redox indicator triphenyl tetrazolium chloride (TTC) is colorless but becomes deep red when reduced [32]. Phage transduction was used to generate a knockout of the promotor for arabinose isomerase (MSB1: HME63: $\triangle \operatorname{araBAD}$ ), inhibiting hydrolysis of arabinose [33]. When grown on agar plates containing both TTC and the reducing sugar arabinose, MSB1 produces dark red colonies, allowing for differentiation of a mixed population of the control strain $\left(\mathrm{ara}^{-}\right)$and a test strain $\left(\mathrm{Cra}^{+}\right)$ based on color (Fig. 3A).

Co-culturing MSB1 with $d n a B: W T$ (or any of the $d n a B$ mutant stains) allowed the monitoring of the competitive growth and fitness in a red:white competition assay. As a control, the mixed population of MSB1(red):dnaB:WT(white) remained constant over 48 hours (Fig. 3B\&C). However, each of the $d n a B$ mutant strains declined in population to near zero in favor of MSB1 within 48 hours. Using the rate of decline overtime, we calculated the selection rate of each strain (Eq. 2) compared to MSB1 [34]. This is an inverse measure of strain fitness, with zero indicating equal fitness to the control strain, as is the case for competition 

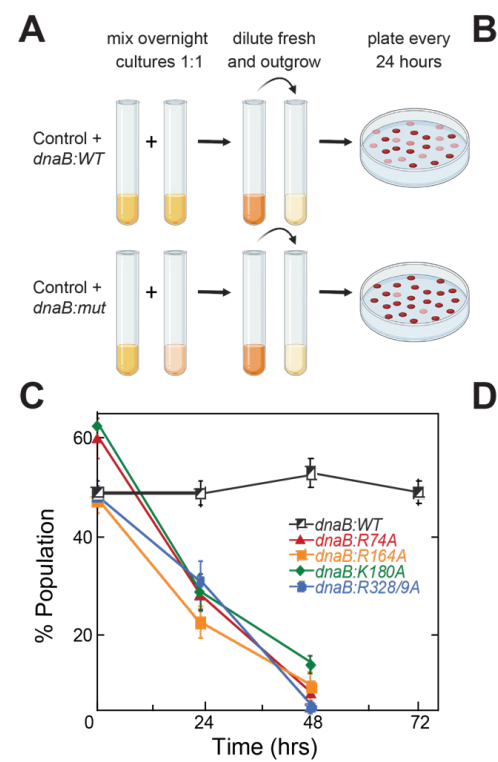
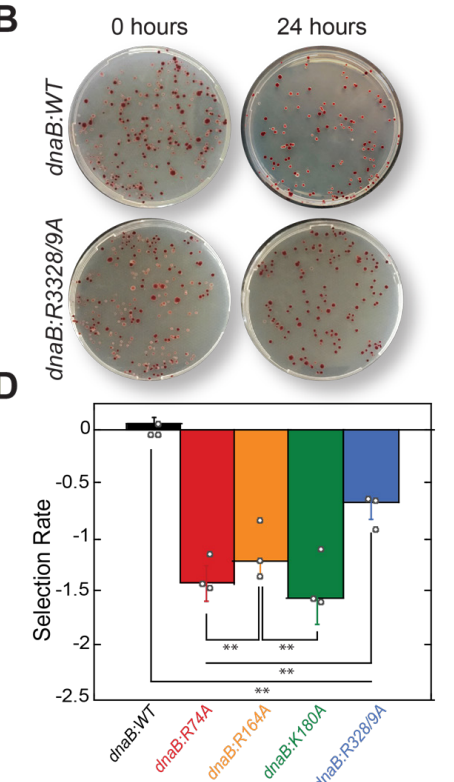

Fig. 3: dnaB mutations reduce strain efficacy. (A) Schematic for a red-white bacterial competition assay, where strains are color coded for colorimetric differentiation over time. Red is the control strain MSB1, and white are the test strains. (B) Representative plates showing changes in population overtime for the equally mixed populations MSB1 versus $d n a B: W T$ or $d n a B: R 328 / 9 A$ at 0 and 24 hours. (C) Changes in population of mixed cultures are plotted over time for $\mathrm{n}=$ 3 biological replicates with error bars representing the standard deviation. (D) The mean selection rate for each strain based on the population change during the first 24 hours (Equation 3). Individual data is presented with open circles. Black bars indicate statistically significant differences. Selection rate is a relative determination of strain fitness compared to the control strain, MSB1. Error bars represent $\pm \mathrm{SD}$ and are within symbols where not visible. Black bars indicate statistically significant differences, where p-values are $*<0.05$ and $* *<0.01$.

with $d n a B: W T$ strains (Fig. 3D). All of the $d n a B$ mutant strains were significantly outcompeted by MSB1 demonstrating a severely decreased strain efficacy. This includes $d n a B: R 74 A$, which competed poorly against the control strain with a selection rate of $-1.34 \pm 0.14$ despite exhibiting near normal exponential growth in Fig. 2B. dnaB:R328/9A had the most competitive selection rate of the mutants with a value of $-0.74 \pm 0.16$, followed by $\operatorname{dnaB}: R 164 \mathrm{~A}$ with a $-1.13 \pm 0.27$ selection rate relative to MSB1. dnaB:K180A was the lease competitive with a selection rate of $1.6 \pm 0.27$, correlating with its slower growth rate in Fig. 2 B.

Helicase dysregulation increased chromosome complexity. To further elucidate how our helicase mutations affect replication cycles, quantitative fluorescence activated cell sorting (FACS) was performed on log phase cells utilizing rifampicin to inhibit transcription and allow for the completion (or 'run-out') of DNA synthesis to observe distinct chromosome densities (Fig. 4A) and cell populations (S3 and S4 Figs.) $[35,36]$. Relative to the parental strain, all mutants had marked increases in chromosome density, correlating with the elongated and filamented cells observed by microscopy for every strain except for $d n a B: R 74 A$ (Fig 2C). Like most actively replicating bacteria, $d n a B: W T$ had major peaks representing 2 and 4 chromosome integers during this 4 hour run-out, confirmed by comparison to a control single chromosome control strain, dnaA(ts) (S5 Fig.). dnaB:R164A has an increase in nucleotide density, with major peaks between 2 and 4 , and 4 and 8 chromosomes, respectively, indicating an odd number of chromosomes. dnaB:K180A has a major chromosome peak of 4 , with a secondary peak at 8 but with diffuse signal in between. dnaB:R74A has major chromosome peaks just to the right of the 4 and 8 integer markers and aligning with major peaks

in $d n a B: R 164 A$, indicating an increase in odd number nucleotide density despite maintaining normal cell size (Fig. 2C). dnaB:R328/9A has the greatest and broadest increase in chromosome density, with a single major peak past 8 chromosome and even more signal to the right.

While the primary peaks represent integer numbers of chromosomes, significant broadening of peaks and loss of definition are seen for several of the strains, especially with the highest nucleotide density. This broadening at the 4 and 8 integer marks for $d n a B: K 180 A$ and the indistinct right-shouldered single peak seen for $d n a B: R 3288 / 9 A$ represent a subpopulation of cells that were unable to complete replication during the substantial 4-hour 'run-out' period. As E. coli grown at $32{ }^{\circ} \mathrm{C}$ can take up to 60 minutes to replicate their entire genome [37], these partially replicated chromosomes are indicative of significant blocks to DNA replication, termination, and/or chromosome segregation. The intermediate odd numbered peaks observed for $d n a B: R 74 A$ and dnaB:R164A further suggest issues of replication stress and are a hallmark of asynchronous initiation.

To further elucidate route causes to increased and asynchronous chromosome numbers, we next performed a qPCR analysis, comparing the relative abundance of the origin (ori) and termination (ter) regions of the chromosome (Fig. 4B). In E. coli, log phase cells generally have an ori:ter ratio $\sim 3$ [38], similar to our control strain $(3.1 \pm 0.4)$. Notably, dnaB:R328/9A, which had the highest and least distinct FACS chromosome signal also had an ori:ter ratio of $4.6 \pm 0.3$, significantly more than any of the other strains. Similarly, dnaB:K180A had a qPCR ori:ter ratio of $4.1 \pm 0.3$, and $d n a B: R 164 A$ had an ori:ter ratio of $3.8 \pm$ 0.2 , both significantly higher than that of $\operatorname{dnaB}: W T$. dnaB:R74A had an ori:ter ratio of $3.3 \pm 0.2$, which was not significantly different than that of the control.

DnaB (R328/9A) is loaded onto ssDNA less efficiently. Previously, it was shown that a constricted DnaB is loaded less efficiently than WT and is generally unable to support rolling circle in vitro DNA synthesis [25]. To test whether there were any perturbations in DnaC dependent loading of these mutant DnaBs onto M13 ssDNA to indirectly explain differences in ori:ter ratios, we utilized a size exclusion chromatography loading assay [26]. Only those DnaB hexamers that are stably loaded on to M13 DNA would shift their elution profile toward the void volume (Fig. 5A and S7 Fig.). DnaB loading was monitored directly

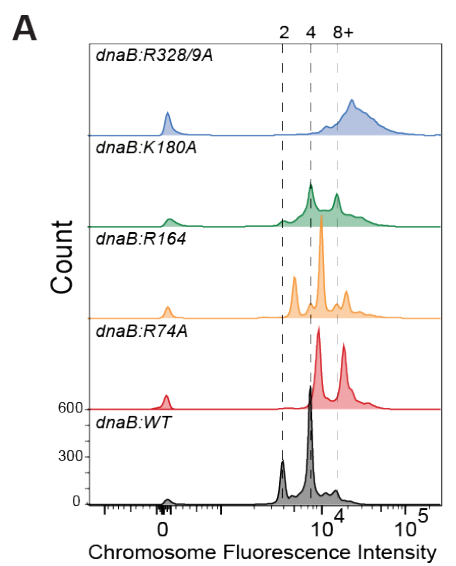

B

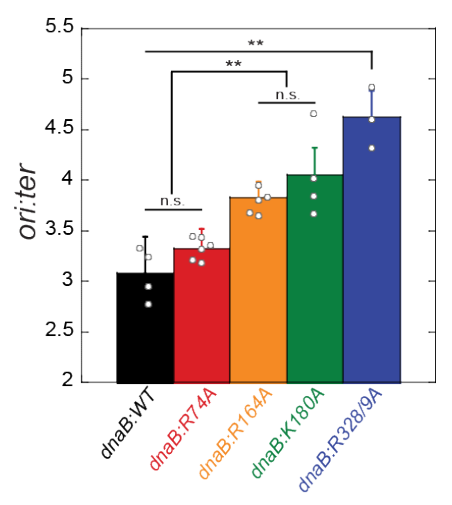

Fig. 4: dnaB:muts show changes in chromosome complexity. (A) Chromosome density was measured by flow cytometry (FACS) in log phase rifampicin 'runout' cultures stained with Sytox green $(n=10,000$ events). Chromosome integers are indicated at the top of the graph. (B) The ori:ter fold-difference measured by qPCR was calculated using the $2^{-\Delta \Delta C t}$ method. Individual data is presented with open circles. Black bars indicate statistically significant differences. Error bars represent $\pm \mathrm{CV}$; Black bars indicate statistically significant differences where $\mathrm{p}$ values are $* *<0.01$. n.s. is not significant. 
A

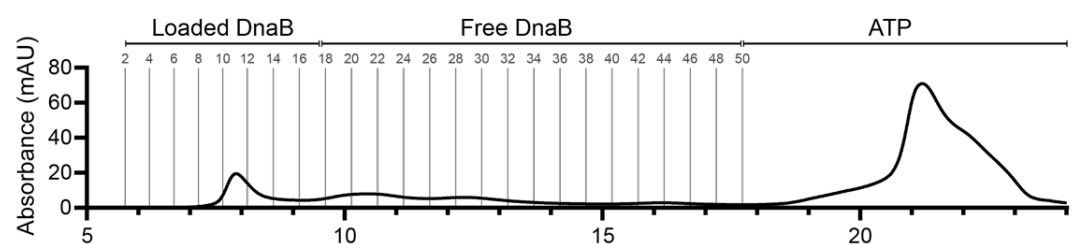

\section{WT DnaB}

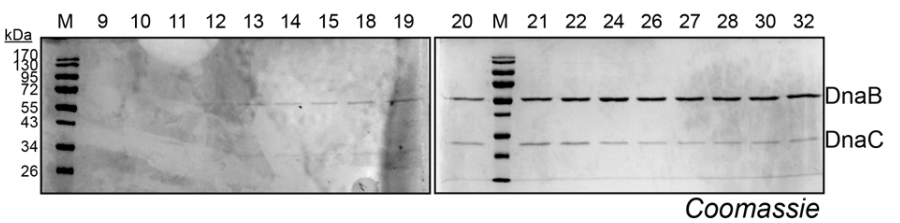

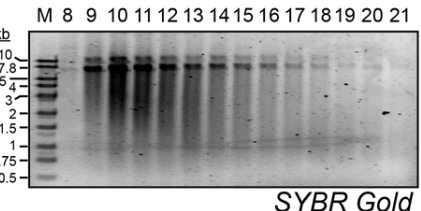

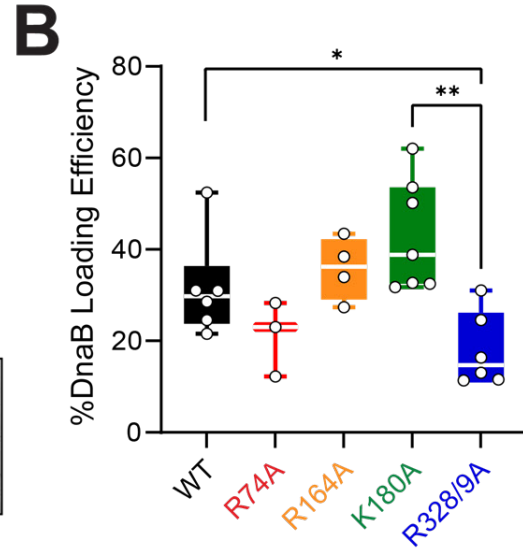

Fig. 5: Size exclusion chromatography DnaB loading assay. (A) DnaB were preincubated with DnaC, M13, and ATP before injecting onto a preequilibrated S200 10/30 size exclusion column according to the Materials and Methods. Example chromatogram and associated SDS-PAGE (Coomassie) and agarose (SYBR-Gold) gels used to monitor loaded DnaB and Free DnaB areas. (B) \%DnaB loading efficiency was calculated according to Equation 4 and all experimental runs plotted (white circles) as box and whiskers for all the DnaBs, where the median is indicated as a while line. Black bars indicate statistically significant differences where p-values are $* *<0.01$ and $*<0.05$

by identifying shifted fractions containing loaded DnaB (and DnaC) by Coomassie stain and M13 DNA by SYBR Gold staining correlating to the chromatogram (A280). Unloaded DnaB/DnaC elute over a range of volumes consistent with several oligomeric conformations. The integrated and isolated peak regions were then used to calculate the $\%$ DNA loading efficiency using ATP as a convenient internal standard to account for any variability in the chromatographic runs according to Equation 4 (Fig. 5B). Although there were some moderate changes in DnaB loading efficiency across the mutants, DnaB (R328/9A) had a significantly reduced loading efficiency compared to WT, $18 \pm 3 \%$ and $32 \pm 4 \%$, respectively. Loading of DnaB (K180A), $43 \pm 5 \%$, is increased slightly over that of WT, but it is not significant at the $95 \%$ confidence level; however, it is significantly different from that of DnaB (R328/9A). Neither DnaB (R74A) or (R164A) loading was significantly different from WT at $21 \pm 5 \%$ and $36 \pm 3 \%$, respectively. Therefore, increased in vitro DnaC dependent loading by DnaB (K180A) may partially explain its increased ori:ter ratio in vivo, however, this is not the case for DnaB (R328/9A) as it has an impaired ability to load onto ssDNA.

dnaB:K180A and dnaB:R328/9A have increased levels of mutagenesis. The poor competitive nature of our helicase mutants coupled with signs of genomic stress naturally led us to investigate their effect on genomic stability. To explore this, we measured the mutation frequency of our $d n a B$ strains by testing for rifampicin resistance (Fig. 6). Briefly, cells were grown in M9 minimal media as an environmental stressor, then exposed to lethal levels of rifampicin [35, 39, 40]. The number of resistant colonies that arose determined the mutation rate. dnaB: K180A has the highest mutation rate at $0.72 \pm 0.17$ mutation events per $10^{6}$ cells, followed by $d n a B: R 328 / 9 A$ with $0.49 \pm 0.01$ events per $10^{6}$ and were both statistically significant compared to the control at 0.12 . There was substantial variability in the frequency of mutations for dnaB: $K 180 \mathrm{~A}$, such that the average rate is not significantly different from dnaB:R328/9A despite the double mutant having a very low deviation. Considering the extremely poor strain viability of $d n a B: K 180 A$, this may suggest a greater spectrum of genetic changes occurring caused by deficiencies in a mutated DnaB. The rates of $d n a B: K 180 A$ and $d n a B: R 328 / 9 A$ are significantly higher than that of $d n a B: W T$, which had a low mutation rate of about $0.12 \pm 0.11$ events per $10^{6}$. Although both dnaB:R74A and dnaB:R164A demonstrated poor fitness (Fig. 2) and increased chromosome number (Fig. 4), neither of these strains showed a mutation rate significantly different to that of the parental strain. Notably, dnaB:K180A had the slowest growth (Fig. 2B) and the lowest selection rate (Fig. 3E) and correlates with the highest mutational frequency out of all the mutant strains.

dnaB:R328/9A has an upregulated SOS response. To determine if the increases in mutagenesis and chromosome complexity correlated with an abundance of mutagenic repair (especially for $d n a B: K 180 \mathrm{~A}$ and dnaB:R328/9A), we investigated whether the SOS response was activated in $d n a B$ mutant strains. The bacterial SOS response is induced when excess ssDNA intermediates are available for RecA polymerization, a common event under DNA damaging conditions, leading to increased expression of mutagenic repair proteins [41, 42]. We measured SOS induction by transforming our strains with a plasmid containing SuperGlo GFP under a RecN SOS-regulated promoter as performed similarly [43]. Strains were grown in the presence of low levels of the crosslinking agent, mitomycin-C (MMC) to heighten any SOS induction differences (Fig. 7).

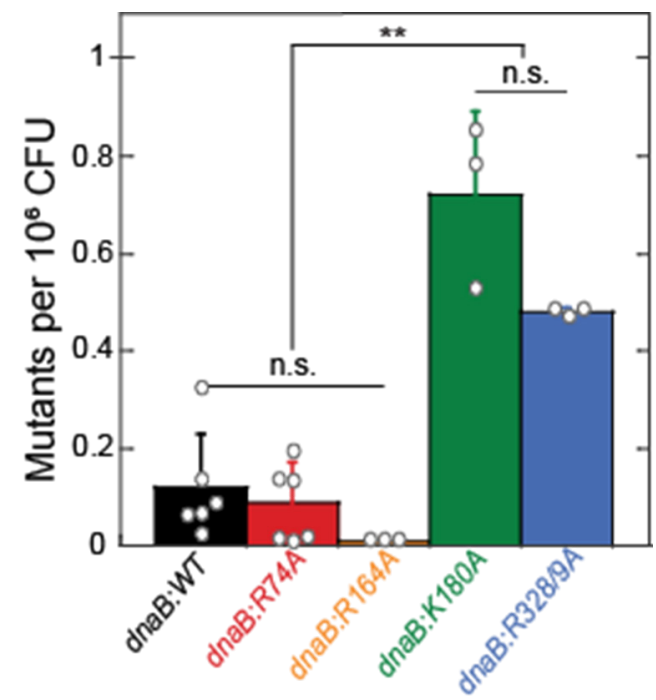

Fig. 6: dnaB:mut reduce DNA stability and increase genomic stress

The mutagenicity of each strain was monitored using a rifampicin resistant assay to quantify number of rif $^{\mathrm{R}}$ colonies after 24-hour growth in minimal media. Average number of resistant colonies per 1 million CFU's are shown (Equation 5). Data is from at least two trials of three technical replicates each $(n \geq 6)$. Error bars represent $\pm \mathrm{SD}$; Black bars indicate statistically significant differences, where p-values are $*<0.05$ and $* *<0.01$. n.s. is not significant. 
A

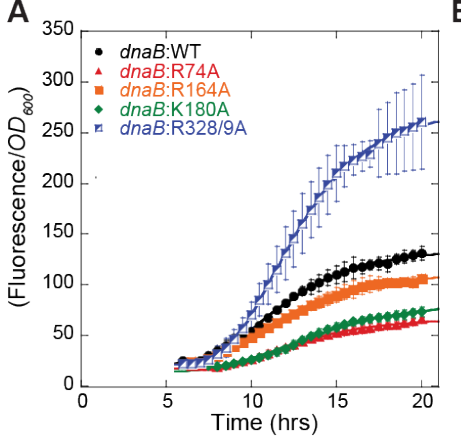

B

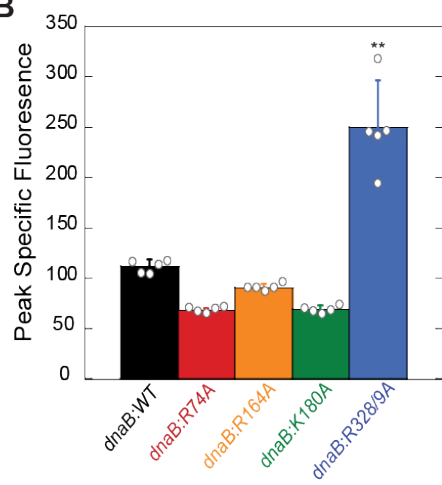

Fig. 7: dnaB:R328/9A induces a greater SOS response. (A) Specific fluorescence for $d n a B$ strains grown with low dose mitomycin $\mathrm{C}(0.001 \mu \mathrm{g} / \mathrm{mL})$ and containing a plasmid expressing GFP under a $r e c N$ promoter to monitor SOS induction. Specific fluorescence is defined as the measured fluorescence divided by the optical density $\left(O D_{600}\right)$ to control for growth. Due to the error inherent in dividing very small numbers, specific fluorescence is not shown for times prior to 5 hours. (B) The maximum fluorescence from $\mathbf{A}$ is plotted for comparison between strains (Eq. 2). Error bars represent \pm SD and are within symbols where not visible. Statistically significant differences are indicated where $\mathrm{p}$-values are $* *<0.01$.

The specific fluorescence (measured by Eq. 6) was fitted with Eq. 2, and the maximum SOS response was quantified for each strain. $d n a B: W T$ had a peak specific fluorescence of $112 \pm 6$; dnaB:R74A had a peak specific fluorescence of $67 \pm 3$; dnaB:R164A had a peak specific fluorescence of $91 \pm 4$; and $d n a B: K 180 A$ had a peak specific fluorescence of $69 \pm 4$. Unlike all other mutants, dnaB:R328/9A had significantly higher SOS induction compared to the parental strain, with a peak specific fluorescence of $250 \pm 46,2.5$-fold greater than the parental strain. SOS induction in dnaB:R328/9A correlates well with elongated cells (Fig. 2), high chromosomal complexity (Fig. 4), increased mutagenesis (Fig. 6), and may be partially explained by decreased DnaB loading (Fig. 5).

A

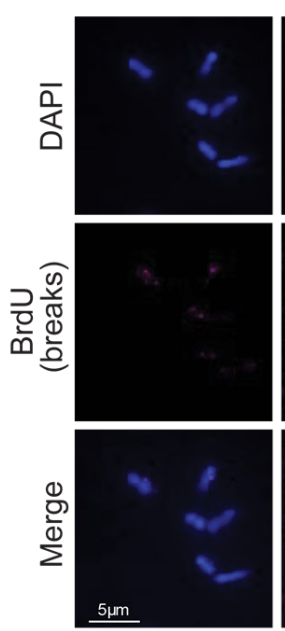

dnaB:WT
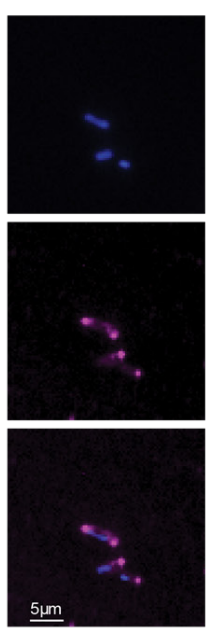

dnaB:R74A
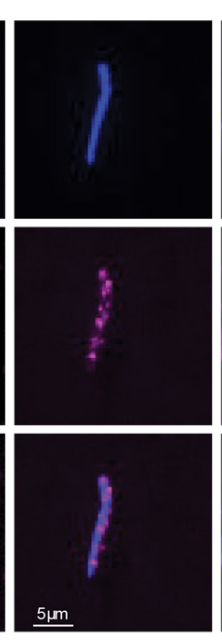

dnaB:R164A
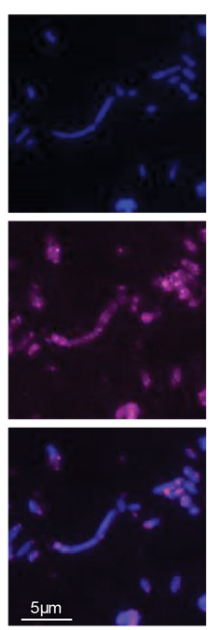

dnaB:K180A
dnaB:mut strains have more DNA strand breaks. To specifically observe and compare differences in DNA damage, we performed a bacterial TUNNEL assay to fluorescently label ssDNA and dsDNA DNA breaks. Log phase cultures were fixed and permeabilized before labeling DNA ends with BrdU and staining the nucleoid with DAPI. Stained cells were imaged, and representative microscopy images are shown (Fig 8A). Interestingly, there was a marked visible increase in BrdU foci for all mutant strains. As a positive control, we exposed a log-phase culture of dnaB:WT to moderate doses of MMC [44], for 45 minutes before harvesting, which also showed significant increases in BrdU fluorescence. Intriguingly, while the other three mutants show widespread and sporadic DNA breaks, the foci in $\operatorname{dnaB}: R 74 A$ were very bright and consistently located at the polls of the cell.

FACS analysis was performed to quantify BrdU abundance for all strains and showed a marked increase for all the $d n a B$ mutants (Fig. 8B). BrdU positive cells were gated at $10^{3}$, with $d n a B: W T$ having $39.4 \%$ of cells with BrdU signal and the positive control (dnaB:WT $+\mathrm{MMC})$ having $88.2 \%$ of cells with BrdU signal. dnaB:R328/9A had $77.1 \%$ of cells with ss and dsDNA breaks, followed by $\operatorname{dnaB}: R 164 A$ with $71.9 \%$, and $d n a B: K 180 A$ with $61.6 \%$. dnaB:R74A had the highest number of cells with ss and dsDNA breaks, with $91.4 \%$ of the population containing BrdU signal, consistent with strong fluorescent signals at the poles in the vast majority of cells (Fig. 8A). Noticeable tailing relative to the parental strain represents populations of cells that have increased DNA and DNA damage (as seen for dnaB:R74A) (S6 Fig.).

\section{$\underline{\text { Discussion }}$}

In this work, we determined that targeted external DnaB surface mutations, previously shown to limit interaction with the excluded strand in vitro also stabilize a constricted hexamer conformation. These mutations, when edited into the bacterial genome, cause widespread genomic and replication stress, alter cellular morphology, and limit cellular fitness. Specifically, dnaB edited strains present with increased DNA damage and mutagenic repair and display signs of replication progression and/or termination defects, even in the absence of any

Fig. 8: dnaB:mut have increased DNA damage under normal conditions. (A) Log phase cells were probed for DNA breaks by a TUNEL assay. Blue (DAPI) represents DNA staining, and pink $(\alpha \mathrm{BrdU})$ represents tagged DNA breaks. $+\mathrm{MMC}$ (pink) in the last panel column are $d n a B: W T$ treated with $0.01 \mu \mathrm{g} / \mathrm{mL} \mathrm{MMC}$. Images shown are representative of the population observed. The fraction of $\alpha$ BrdU labeled cells was (B) measured and quantified by flow cytometry (FACS), utilizing the same log phase cultures as the microscopy images. BrdU-negative and positive populations were gated at $10^{2}$ and the percentages are indicated on the plot; data is from $\mathrm{n}=10,000$ events. 
external genotoxic stress. These findings highlight the functional importance of helicase regulation for faithful and efficient replication.

Based on current research, it is suggested that the helicase conformation plays a role in DNA loading, unwinding efficiency, protein associations at the fork, and helicase-polymerase coupling $[14,25]$. In the event of polymerase pausing or stalling, helicase activity should slow, likely changing conformation to engage with the excluded strand and minimize forward progression [26, 45, 46]. DnaG, which favors the dilated state of DnaB, has been shown to limit replisome progression and generate pausing events [47], consistent with a model where the helicase constricts for efficient unwinding and dilates to slow unwinding and allow for more efficient priming. It is unclear whether the helicase physically decouples from the polymerase during these events, but it is apparent that it continues to generate ssDNA with an approximate 10fold reduction in rate [48]. Our electrostatic SEW mutants stabilize the constricted state and disrupt interaction with the excluded strand [4] and are poised for rapid unwinding, suggesting that their helicase activity should be continuous and fast and independent of Pol activity, possibly to the detriment of cell proliferation and organismal fitness. However, while one function of helicase regulation is clearly to limit ssDNA production, a lack of increased SOS response for most of the mutants indicates that the function of helicase regulation goes beyond limiting unwinding and impacts other aspects of replisome activities.

DnaB(R74A) and (R164A) generally stabilize the constricted state but were more intermediate than the other mutants and had no defects in ssDNA loading. This ability to dilate, even infrequently, likely contributes to the more regular size, stable ori:ter ratio and chromosome density, and low mutagenesis and SOS of the dnaB:R74A strain. However, while $d n a B: R 164 A$ also had low mutation frequency and SOS response, like $d n a B: R 74 A$, it displayed genomic stress markers and generally poor fitness despite having a near identical dilation frequency. This highlights the importance of dilation and excluded strand engagement for normal, damage-resistant replication, while also making it evident that moderate dilation alone is not enough to restore normal or even mostly normal function.

While all of the selected DnaB residues were predicted to be surface exposed based on homology modeling with a G. ste DnaB crystal structure, the two more prominent SEW sites, K180 and R328/9, appear to be partially buried in a more recent crystal structure [26] of a constricted E. coli DnaB (Fig. S8). When mutated, DnaB (K180A or R328/9A) stabilize a fully constricted state. DnaB unwinding activity is

\section{Decoupling}
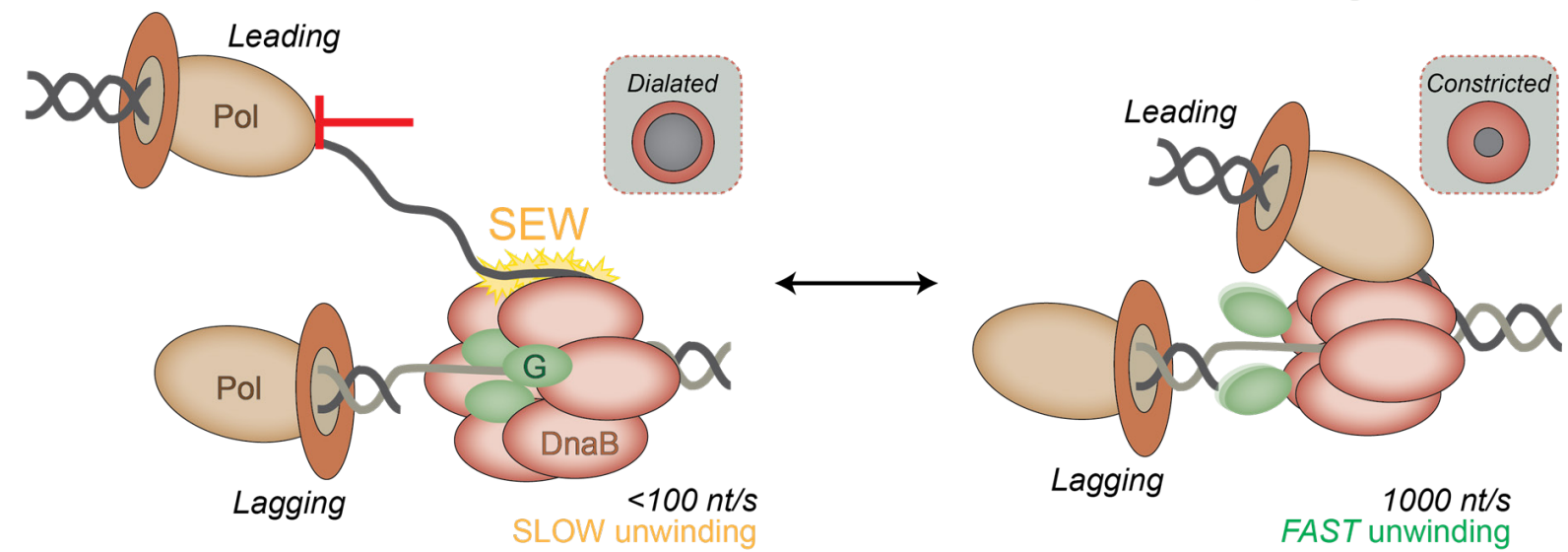

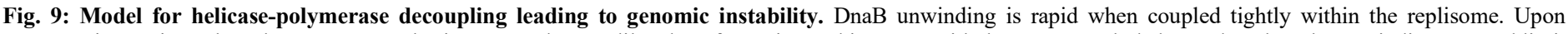

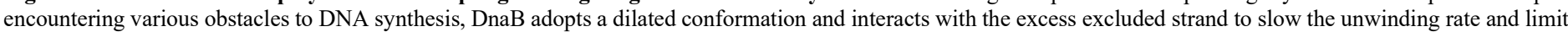

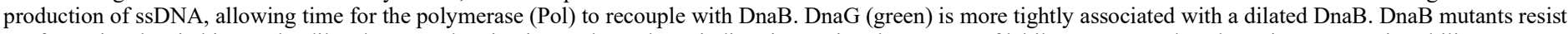
conformational switching to the dilated state and maintain accelerated unwinding, increasing the amount of labile ssDNA produced causing genome instability.

also greatly stimulated by the addition of tau [14], which would be present in an active replisome serving to coordinate polymerase and helicase activity. We therefore suggest that key SEW residues are concealed when DnaB is constricted to allow fast and efficient replisome progression but become exposed in the dilated state to limit helicase activity (Fig. 9). Interestingly, a dilated state of DnaB has a higher affinity with the CLC and may possibly explain the rapid exchange/recruitment of the Pol III core (Pol III*) seen in in vitro assays [49], single-molecule [50], and in vivo imaging experiments [51]. Additionally, we note that both K180 and R328/9 are located in the Cterminal domain (CTD) of the enzyme; R164 is located in the linker helix; and R74 is located in the N-terminal domain (NTD) (Fig. S7). While the N-terminal collar significantly contributes to the helicase conformation $[14,25]$, it is clear that each domain of the helicase plays a role in the conformational dynamics and interactions within the replisome.

Interestingly, mutation of $d n a B: R 74 A$ in the NTD of the protein had the least impact on cellular growth and morphology, with low mutagenesis and SOS, and was the only strain to maintain normal ori:ter ratios. Despite this, it had the highest fraction of cells with BrdU foci, localized primarily at the poles, and a large increase in chromosome number. This polar TUNEL localization was consistent across images and indicates that DNA breaks may be localized to the ori and/or ter regions of the chromosome, which migrate to the ends of the cell during chromosome segregation [52-54]. Although DnaC loading of DnaB (R74A) was not significantly affected, these highly localized breaks suggest specific issues with replication initiation and termination, which may instead be the result of altered interactions with DnaA and/or Tus $[55,56]$. Despite this, dnaB:R74A maintained normal growth rates, cell size, and ori:ter ratio, but decreased fitness compared to the parental strain.

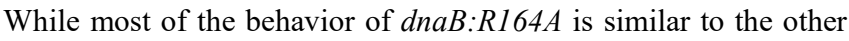
mutants, it was the only strain to have elongated cells even in stationary phase. The R164 residue is in the linker helix domain of DnaB, which is responsible for coordinating the hexamerization of the helicase from monomer into its active form [57] and is responsible for interaction with the primase DnaG [58]. The location of the mutation may contribute to the observed phenotype of this strain. In addition to slow growth, elongated cells, and poor competitiveness which was consistent for most strains, dnaB:R164A exhibited strong asynchronous initiation, as indicated by chromosome numbers other than $2^{\text {n }}$ (but without peak

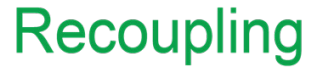


Behrmann et al., 25/05/2021 - preprint copy - BioRxiv

broadening) and a unique cell population with high chromosome complexity but smaller cell size. dnaB:R164A also had widespread ss and dsDNA breaks, similar to $d n a B: K 180 A$ and $d n a B: R 328 / 9 A$. Interestingly, unique BrdU populations were observed for dnaB:R164A when compared to cell size. Specific behavior and contributions of the linker helix to helicase conformation are worth further investigation.

Helicase regulation has been shown vital to maintaining a stable DNA duplex in the event of discontinuous replication. Recent work suggests that rather than a smooth and continuous process, replisome progression is naturally stochastic [59] with frequent pausing and polymerase exchange [51], indicating that decoupling is a natural component of the replication process (Fig. 9). The effect of these dnaB mutations on strain growth and fitness, SOS induction, and chromosome complexity even in the absence of environmental stress or exogenous damage support the notion of frequent replisome pausing and helicasepolymerase decoupling, which if not regulated could lead to ssDNA buildup, lack of replisome coordination, and reduction in fidelity. This agrees with previous observations that proposed helicase progression is a vital fail-safe mechanism to maintain helicase-polymerase coordination during polymerase pausing and allow time for TLS or repair in the event of DNA damage [24]. More binary rapid unwinding kinetics by a constricted helicase cannot adequately respond to stochastic DNA polymerase progression, likely contributing to decoupled synthesis and unwinding activity, production of labile ssDNA, less frequent priming, and challenging DNA structures migrating behind to limit synthesis. These effects compound with initial replication challenges, encouraging slow, inefficient replisome progression and resulting in poor performance as seen with our SEW mutants.

The effect of $d n a B$ mutants on faithful and efficient replisome activity is complex and confirms the importance of helicase dynamics within the replisome as a whole. Disruption of this process, however minor, will amplify with replication and environmental stress in vivo. Replisome cohesion is a critical aspect of the protein complex, as it harnesses many mobile elements that work in stochastic symphony. Helicase conformation and SEW are jointly responsible for regulating not only helicase unwinding activity, but also controlling aspects of replisome coordination (loading, priming, unwinding) and progression (DNA unwinding speed) in the presence of various genomic obstacles. Our data suggests that both speed and helicase-protein interactions contribute to the deleterious effects seen for DnaB malfunction in vivo, and further studies are needed to elucidate the specific role and impact of the helicase-polymerase tether, the CLC, and whether decoupling occurs at sites of damage.

\section{$\underline{\text { Materials and Methods }}$}

\section{Purification of E. coli DnaB and mutants}

Wild-type E. coli DnaB and mutants (R74A, R164A, K180A, R328/329A) were independently expressed in C41 strain (Lucigen, Middelton, WI) from pET11bderived plasmids as previously described [4]. Briefly, IPTG $(1 \mathrm{mM})$ induced DnaB was pelleted and resuspended in the lysis buffer $(20 \mathrm{mM}$ Tris $[\mathrm{pH} 7.5], 500 \mathrm{mM}$ $\mathrm{NaCl}, 10 \%$ glycerol, $10 \mathrm{mM} \mathrm{MgCl} 2,1 \mathrm{mM}$ PMSF) and lysed using lysozyme and sonication. Ammonium sulfate $(0.17 \mathrm{~g} / \mathrm{ml})$ was added to the resultant supernatant, pelleted, and then resuspended in Buffer A (20 mM Tris [pH 7.5], $10 \mathrm{mM} \mathrm{MgCl}$, $0.1 \mathrm{M} \mathrm{NaCl}, 10 \%$ glycerol, $0.01 \mathrm{mM}$ ATP, $1 \mathrm{mM}$ BME) and applied to HiTrap MonoQ column (Cytiva, Marlborough, MA) and eluted with a stepwise gradient of buffer A supplemented with $0.75 \mathrm{M} \mathrm{NaCl}$. The fractions were combined and applied to Superdex S-200 26/600 gel filtration column (Cytiva, Marlborough, MA) with Buffer B (20 mM Tris [pH 7.5], $5 \mathrm{mM} \mathrm{MgCl}_{2}, 0.8 \mathrm{M} \mathrm{NaCl}, 10 \%$ glycerol, $0.1 \mathrm{mM}$ ATP, $1 \mathrm{mM}$ BME). Combined peak fractions were concentrated and dialyzed against storage buffer $(20 \mathrm{mM}$ Tris [pH 8.5], $500 \mathrm{mM} \mathrm{NaCl}, 5 \mathrm{mM}$ DTT, $50 \%$ glycerol).

\section{DNA Translocation Assay}

The translocation assay was performed as described [14] in a 96-well plate (Corning) using a Varioskan Lux Microplate Reader (Thermo Scientific,
Waltham, MA). Substrates (S1 Table) were purchased from Integrated DNA Technologies (IDT, Coralville, IA). Annealing reactions were performed in a solution of $10 \mathrm{mM}$ Tris-HCL, $1 \mathrm{mM}$ EDTA, and $100 \mathrm{mM} \mathrm{NaCl}(\mathrm{pH} 7.5)$ using a thermocycler following the protocol $95^{\circ} \mathrm{C}$ for $6 \mathrm{~min}$, then decreases $1{ }^{\circ} \mathrm{C} / \mathrm{min}$ with a final hold at $25^{\circ} \mathrm{C}$. Translocation reactions contained $20 \mathrm{nM}$ substrate and 40 $\mathrm{nM}$ DnaB hexamer in reaction buffer (20 mM HEPES-KOH [pH 7.5], $5 \mathrm{mM}$ magnesium acetate, $50 \mathrm{mM}$ potassium glutamate, $5 \%$ glycerol, $0.2 \mathrm{mg} / \mathrm{mL}$ BSA, $4 \mathrm{mM}$ DTT, ), and initiated with $1 \mathrm{mM}$ ATP and $200 \mathrm{nM}$ trap (unlabeled DNA165) (S1 Table). Data was fit to the following equation for a single exponential:

$$
\% D N A \text { unwound }=A_{0}+A e^{-k t}
$$

where $A_{0}$ is the lower asymptote, $A$ is the amplitude, $k$ is rate and $t$ is time.

\section{CRISPR-Cas9 Genomic Editing}

Using a dual-plasmid system designed for E. coli engineering [60], we created four distinct strains that carry precise mutations in the gene (dnaB) that codes for the replicative helicase DnaB (S1 Fig. and S1 Table). The parental strain, HME63, is a derivative of $E$. coli $\mathrm{W} 3110$ with $\Delta m u t S$ for suppression of mismatch repair and optimized for recombineering by the expression of $\lambda$-red genes (gam, exo, and bet) under control of a temperature sensitive (ts) repressor [61]. All mutant dna $B$ strains are derivatives of HME63, created using dual plasmid CRISPR-Cas9 system to generate a point mutation in the DnaB helicase enzyme by chromosomal DNA modification [60,62-64]. Briefly, 30 base regions of the dnaB gene (S1 Table) targeting the mutation site were inserted into the pCRISPR plasmid (Addgene: 42875) as a guide RNA (gRNA) and electroporated into HME63 already containing pCas9 (Addgene: 42876 ). The $d n a B$ gRNA was designed to be centered on the desired mutation site and at the closest adjacent PAM sequence (5'-NGG). $1 \mu \mathrm{M}$ of a synthetic 60 base ssDNA oligonucleotide template for homologous recombination (HR) containing the desired mutation on the lagging strand was simultaneously electroporated with $100 \mathrm{ng}$ pCRISPR to edit the bacterial genome (S1 Table). HME63 electroporated with pCRISPR and pCas9 but without the editing oligonucleotide were used to determine background levels. Precise genome editing was monitored using PCR and screening for a novel engineered restriction site (S1 Fig.) before confirmation by DNA sequencing of the locus (UT Austin, Genome Sequencing and Analysis Facility). All confirmed strains were immediately frozen as stocks, then outgrown in the absence of Kan/Chl to remove CRISPR-Cas9 editing plasmids, and then reconfirmed for the $d n a B$ mutation by PCR and restriction digest. All subsequent cultures were grown in LB supplemented with $100 \mu \mathrm{g} / \mathrm{mL}$ ampicillin, and all incubation steps were performed at $32{ }^{\circ} \mathrm{C}$, unless otherwise stated. All strains are listed in S2 Table.

\section{Growth Curves}

Growth curves were recorded by diluting overnight clonal cultures to $\mathrm{OD}_{600} \sim 0.01$ in LB and aliquoting $200 \mu \mathrm{L}$ into a black-walled clear-bottomed 96-well plate (Corning). The cultures were incubated at $32{ }^{\circ} \mathrm{C}$ with aeration at $225 \mathrm{RPM}$ and the $\mathrm{OD}_{600}$ was recorded at 30 -minute intervals using a Varioskan Lux microplate reader equipped with SkanIt 5.0 software (Thermo Scientific, Waltham, MA). Data was processed using KaleidaGraph (Synergy Software, v.4.5.3) and fit to a modified 4-parameter Gompertz growth model [65] according to the following equation:

$$
w(t)=B+A^{-e^{\left.-\frac{k_{\mathrm{z}} \times 2.7182}{A} \times\left(T_{\text {lag }}-t\right)+1\right)}}
$$

where $w(t)$ is the density as a function of time, $B$ is the lower asymptote, $A$ is the upper asymptote, $K_{z}$ is the absolute growth rate, $T_{\text {lag }}$ is the lag time of the culture, and $t$ is time.

\section{Microscopy}

All microscopy images were obtained using an Olympus Brightfield Microscope IX-81 (Olympus Corp., Center Valley, PA) using a 60x objective with oil immersion. For stationary phase cells, $1 \mathrm{~mL}$ of each culture was grown overnight in LB/Amp at $32{ }^{\circ} \mathrm{C}$, pelleted, washed in PBS, and then fixed with $70 \%$ ethanol. 2 $\mu \mathrm{L}$ of the fixed sample was spotted onto a microscope slide and allowed to dry. DAPI (ThermoFisher, Waltham, MA) was added to mounting media $(2.5 \%$ DABCO, $90 \%$ glycerol, $7.5 \%$ PBS) to create a dual staining and mounting solution. $3 \mu \mathrm{L}$ of this solution was added to cover the fixed cells, then immediately topped with a coverslip and sealed with clear polish. Slides were stored at $4{ }^{\circ} \mathrm{C}$ in the dark overnight prior to imaging. For DAPI-only stained log phase cells, overnight cultures were diluted 1:1000 in LB and grown with aeration until OD $0.2-0.4$ before following the same procedure described for stationary phase cells. Filamentation was quantified by blinded counting and measuring $\geq 200$ cells for each condition using Image J [66]. This data was analyzed by excel and plotted using Prism 9.1 (GraphPad, San Diego CA).

\section{Red-white Strain Competition Assays}

The strain fitness was determined using a red-white assay as described [29]. Construction of the control strain was done using P1 phage transduction. The phage was harvested from the cell line EAW214, a derivative of MG1655 engineered to contain a mutant FRT-KanR-wtFRT cassette in place of the araBAD 
promotor [29]. Through transduction, the neutral $\triangle a r a B A D$ mutation was transferred to the parental strain (HME63) and designated MSB1

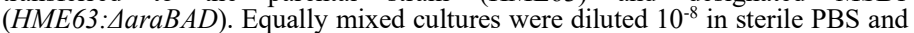
plated onto tetrazolium arabinose (TA) indicator plates, containing $0.2 \mathrm{mg} / \mathrm{mL}$ triphenyl tetrazolium chloride (Sigma, St. Louis, MO) and 1\% arabinose (Oakwood Products, Estill, SC), and then grown at $32{ }^{\circ} \mathrm{C}$ for $24-36$ hours before colonies were counted and sorted by color. MSB1 will have red color when grown on TA plates and allows for easy colorimetric differentiation between the dnaB mutants $\left(\mathrm{ara}^{+}\right)$strains. Mixed cultures were sampled every 24 hours until a strong divergence in population was seen or 72 hours was reached. Relative selection rate was calculated according to the following equation:

$$
\text { selection rate } / \text { day }=(r)=\ln \frac{A 1}{A 0}-\ln \frac{B 1}{B 0}
$$

where $A_{0}$ and $B_{0}$ are the CFU fraction of strains $\mathrm{A}$ and $\mathrm{B}$ at time 0 , and $A_{l}$ and $B_{l}$ are the CFU fraction of strains A and B after 24 hours.

\section{Flow Cytometry}

To quantify cell size and chromosome number, overnight cultures were diluted and grown until mid-exponential phase $\left(\mathrm{OD}_{600} \sim 0.3\right)$ before treatment with 150 $\mathrm{mg} / \mathrm{mL}$ rifampicin and $10 \mathrm{mg} / \mathrm{mL}$ cephalexin (TCI America, Portland, OR). Samples were then incubated with shaking for 4 hours to allow for completion of chromosome synthesis (or 'run out'). Samples were pelleted, washed with cold TE buffer, and then fixed in $70 \%$ ethanol. Fixed cells were pelleted, washed in filtered PBS, and then resuspended in 500-1000 $\mu \mathrm{L}$ TBS containing 1.5 $\mu \mathrm{M}$ Sytox Green (Invitrogen, Carlsbad, CA) and $50 \mu \mathrm{g} / \mathrm{mL}$ RNaseA for 30 minutes at $4{ }^{\circ} \mathrm{C}$ in the dark. Stained samples were pelleted and then resuspended in sterile PBS before analysis using a FACSverse (BD Biosciences, San Jose, CA). The parental strain was used as a chromosome control; run-out of normal actively replicating cells gives major peaks for 2 and 4 chromosomes used as reference for chromosomal number. A control experiment comparing the parental strain with CM742 containing a ts mutant of $d n a A$ (S1 Table) [67] to confirm chromosome numbers was performed (S5 Fig.). To prepare, an overnight culture of CM742 was diluted and grown to mid-exponential phase $\left(\mathrm{OD}_{600} \sim 0.3\right)$ before treatment with 150 $\mathrm{mg} / \mathrm{mL}$ rifampicin and $10 \mathrm{mg} / \mathrm{mL}$ cephalexin, and then incubated at $42^{\circ} \mathrm{C}$ for 2 hours before harvesting as described above. Data was plotted and presented using FloJo software (BD Biosciences).

Quantification of DNA breaks was performed using the TUNEL assay described above and quantified using FACS. Antibody-stained cultures were pelleted, resuspended in $1 \mathrm{~mL}$ sheath fluid (sterile $1 \mathrm{x}$ PBS) with $1.5 \mu \mathrm{M}$ Sytox Green, and incubated in the dark for 30 minutes. Samples were diluted with an additional $1 \mathrm{~mL}$ sheath fluid before being analyzed by FACSverse. HME63 exposed to $0.01 \mu \mathrm{g} / \mathrm{mL}$ MMC prior to harvesting was used as a positive control; Sytox green alone and fluorescently labeled BrdU alone were used as signal controls. Data was plotted and quantified using FloJo software.

Fraction of live versus dead cells was determined by diluting overnight cultures and growing until mid-exponential phase $\left(\mathrm{OD}_{600} \sim 0.3\right)$ before samples were pelleted, washed with cold TE buffer (Tris-EDTA), and then resuspended in $100 \mu \mathrm{L}$ cold TE with $2 \mu \mathrm{g} / \mathrm{mL}$ propidium iodide (PI) (ThermoFisher, Waltham, MA). After 30 -minute incubation at $4{ }^{\circ} \mathrm{C}$ in the dark, stained samples were pelleted, washed in filtered PBS, and then resuspended in cold 500-1000 $\mu$ L PBS. Positive control was fixed with ethanol prior to PI staining, and negative controls were not exposed to PI. Samples were analyzed using a FACSverse, and the data was plotted using FloJo software.

\section{$q P C R$}

To determine the relative chromosomal complexity for the mutant and parental strains, quantitative PCR was performed using a Quant-Studio 6 Flex Real-Time PCR instrument (Thermo Scientific, Waltham, MA). For sample preparation, overnight cultures were diluted 1:1000 in $3 \mathrm{~mL} \mathrm{LB}$, grown at $32^{\circ} \mathrm{C}$ until $\mathrm{OD} \sim 0.5$ -0.85 , and then pelleted and fixed in $70 \%$ ethanol. Harvested cultures were kept at $4{ }^{\circ} \mathrm{C}$. Before use, fixed cells were washed and resuspended in $1 \mathrm{~mL}$ sterile water. The qPCR assay was performed using a PowerUp SYBR green master mix (Applied Biosciences, Beverly Hills, CA), $1 \mu \mathrm{L}$ resuspended cells, and $0.5 \mu \mathrm{L}$ each of $100 \mu \mathrm{M}$ forward and reverse primers (S1 Table). The ori:ter $\mathrm{qPCR}$ ratio was calculated using the $2^{-\Delta \Lambda \mathrm{Ct}}$ method for comparative cycle threshold $(\mathrm{Ct})$ analysis $[38,68]$. A fixed overnight sample of the parental strain, where the population would have an ori:ter $\sim 1$, was used for normalization in every run. Each sample had a minimum of five technical replicates in each cycling run, and the mean $\mathrm{Ct}$ value was used to calculate the ori:ter ratio.

\section{DnaB Loading Assay}

Purified E. coli DnaB WT and mutants ( $3 \mu \mathrm{M}$ hexamer) and E. coli DnaC $(18 \mu \mathrm{M}$ monomer) were mixed with $11 \mu \mathrm{g}$ of M13 ssDNA (Guild Biosciences, Dublin, $\mathrm{OH}$ ) to a final volume of $250 \mu \mathrm{L}$ in the presence of $20 \mathrm{mM}$ Tris $\mathrm{pH} 8.5,200 \mathrm{mM}$ $\mathrm{NaCl}, 5 \%$ glycerol, $5 \mathrm{mM} \mathrm{MgCl}_{2}, 5 \mathrm{mM}$ ATP. Reactions were incubated for 10 min at $37^{\circ} \mathrm{C}$ and then applied to a Superdex $20010 / 300$ size exclusion column equilibrated in the same buffer minus ATP using AKTA Pure 25L (Cytiva, Marlborough, MA). $250 \mu \mathrm{L}$ fractions were collected and representative fractions were resolved on both $15 \%$ SDS-PAGE and $1 \%$ agarose gels. The gels were stained with either Coomassie or 1X SYBR Gold (Thermo Scientific, Waltham, MA), respectively, imaged using Gel Doc EZ gel documentation system (BioRad), and analyzed to determine the fractions containing both DnaB and ssDNA. The area $\left(\mathrm{ml}^{*} \mathrm{mAu}\right)$ under the chromatographic curve was integrated for the separate regions from the elution profile containing: 1) DnaB loaded onto M13 ssDNA $\left.\left(\mathrm{DNA}^{\mathrm{L}}\right), 2\right)$ unloaded combinations of DnaB and DnaC $\left(\mathrm{DNA}^{\mathrm{F}}\right)$, and 3) ATP alone using Unicorn 7.1 (Cytiva, Marlborough, MA). The DnaB loading efficiency was calculated as a ratio of the loaded DnaB area over that of the total DnaB area normalized to the area of the ATP peak as an internal standard according to:

$$
\% \text { DnaB Loaded }=\left(\frac{\frac{D n a B^{L}}{A T P}-\frac{M 13}{A T P}}{\frac{D n a B^{L}}{A T P}+\frac{D n a B^{F}}{A T P}}\right) \times 100
$$

The $\mathrm{A}_{280}$ signal contributed by M13 alone (in a separate chromatograph run without protein) was subtracted from peak 1 again using ATP as a normalized internal standard. The experimental data from at least three independent experiments were averaged, plotted as a box and whiskers, and analyzed for significant differences from WT DnaB using parametric t-tests in Prism 9.1 (GraphPad, San Diego CA).

\section{Strain Mutagenesis Assay}

To determine the mutation frequency of each strain, a rifampicin resistant assay was performed as previously described [69]. To test the base mutagenesis rate, fresh overnight cultures were subcultured 1:1000 in M9 minimal media supplemented with $0.2 \%$ glucose, $5 \mu \mathrm{g} / \mathrm{mL}$ thiamine (Acros Organics, Waltham, MA), and $0.4 \%$ casamino acids (Fisher Bioreagents, Waltham, MA), and allowed to outgrow for 24 hours before spreading onto plates containing $50 \mu \mathrm{g} / \mathrm{mL}$ rifampicin (Rif) (ThermoFisher, Waltham, MA). Overnight cultures were subcultured in $\mathrm{M} 9$ until $\mathrm{OD}_{600} \sim 0.4$, then cultures were pelleted and washed with PBS before resuspension in M9 and grown overnight with shaking. Identical aliquots were plated onto Rif + , and diluted and plated onto Rif- plates for colonyforming unit (CFU) controls. All incubation and shaking steps were performed at $32{ }^{\circ} \mathrm{C}$. Mutation frequency was calculated as the ratio of mutants to total CFUs as follows:

$$
\frac{A}{B \times 10^{8}}=\text { mutation frequency }
$$

where $A$ is the number of mutant CFUs (colonies on the Rif + plate), $B$ is the number of total CFUs (colonies on the Rif- plate), and $10^{8}$ is the dilution factor for $B$

\section{SOS induction assay}

Mutant and parental strains were transformed with the plasmid pEAW915, which contains SuperGlo GFP (Qbiogene) under the control of the E. coli recN promoter, in the plasmid pACYC184 [43]. Successfully transformed cells were selected by Kanamycin resistance. SOS fluorescence curves were recorded by diluting overnight clonal cultures 1:1000 in LB with $0.001 \mu \mathrm{g} / \mathrm{mL}$ MMC (Thermo Fisher, Waltham, MA) and aliquoting $250 \mu \mathrm{L}$ into a white clear-bottomed 96 -well plate (Corning). The cultures were incubated at $32^{\circ} \mathrm{C}$ with aeration at $225 \mathrm{RPM}$ and the both the $\mathrm{OD}_{600}$ and fluorescence $(474 \mathrm{~nm}$ excitation / $509 \mathrm{~nm}$ emission) were recorded at 30-minute intervals using a Varioskan Lux multi-mode microplate reader equipped with SkanIt 5.0 software (Thermo Scientific, Waltham, MA) Specific fluorescence was determined by dividing the fluorescence by the absorbance to control for population density using the following equation:

$$
\text { Spec Fluor }=\frac{\text { fluoresence }(509 \mathrm{~nm})}{\text { absorbance }\left(O D_{600}\right)}
$$

Data was processed using KaleidaGraph (Synergy Software, v.4.5.3) and fit to Equation 2 for quantification of the upper asymptote.

\section{Terminal deoxyribonucleotide transferase-mediated dUTP nick end labeling (TUNEL) assay}

For microscopic imaging of DNA breaks, log phase cultures were harvested at OD $\sim 0.3$ by pelleting and washing PBS, then fixed in $1 \mathrm{~mL}$ of ice cold formaldehyde solution (4\% paraformaldehyde in 1x PBS) as described [70]. Cells were incubated in fixing solution for 30 minutes at room temperature, pelleted, and washed with PBS. As a positive control, log phase HME63 was exposed to $0.01 \mu \mathrm{g} / \mathrm{ml}$ MMC (Thermo Fisher, Waltham, MA) for 60 minutes prior to harvesting. After fixation, cells were resuspended in $500 \mu \mathrm{L}$ ice cold permeabilization solution $(0.1 \%$ Triton $\mathrm{X}-100$ and $0.1 \%$ sodium citrate) and incubated on ice for 2 minutes. Cells were again pelleted, washed, resuspended in PBS, and stored at $4{ }^{\circ} \mathrm{C}$ overnight.

dUTP was added to DNA ends by pelleting stored cells and resuspending in $100 \mu \mathrm{L}$ of elongation buffer (1X terminal deoxytransferase [TdT] buffer, $2.5 \mathrm{mM}$ $\mathrm{CoCl}_{2}, 0.1 \mathrm{mM} \mathrm{BrdU}, 5 \mathrm{U}$ of TdT [Thermo-Fisher, Waltham, MA]) and incubating at $37^{\circ} \mathrm{C}$ for $60 \mathrm{~min}$. After elongation, cells were pelleted, washed with PBS, and then resuspended in blocking solution (4\% BSA in 1X TBST) for 30 minutes at room temperature. To fluorescently label BrdU labelled ends, blocked cells were pelleted, washed with blocking solution, and resuspended in $100 \mu \mathrm{L}$ of primary antibody solution (1:100 mouse- $\alpha-B r d U$ [BD Bioscience, Franklin Lakes, NJ] in 
Behrmann et al., 25/05/2021 - preprint copy - BioRxiv

TBST with 4\% BSA) for 60 minutes at room temperature. Afterwards, cells were pelleted and washed with blocking solution, resuspended in $100 \mu \mathrm{L}$ of secondary antibody (1:500 $\alpha$-mouse IgG-Alexa647 [ThermoFisher, Waltham, MA] in TBST with $4 \%$ BSA), and incubated in the dark for 60 minutes at room temperature. Cells were pelleted again, washed once with TBST, then washed with and resuspended in PBS before mounting using DABCO-DAPI solution. Slides were stored at $4{ }^{\circ} \mathrm{C}$ overnight and then imaged by epifluorescence microscopy as described above.

\section{End Matter}

\section{Author Contributions and Notes}

MSB: conceptualization, formal analysis, investigation, validation, visualization, project administration, data curation, methodology, writing - original draft, writing - review and editing; HMP: investigation, writing - review and editing; JMH: investigation; TAV: investigation; MAT: conceptualization, formal analysis, investigation, visualization, methodology, supervision, funding acquisition, project administration, resources, writing - original draft, writingreview and editing

The authors declare no conflict of interest.

This article contains supporting information online.

\section{Acknowledgments}

Special thanks to Michael Cox and Elizabeth Wood (UW-Madison) for helpful advice and for providing us with bacterial strains and plasmids and to the Court lab for generously providing strains for the genome editing. We thank Preston Jones for aiding in developing the bacterial editing of $\operatorname{dnaB}$. We also thank Susan Rosenberg, Jun Xia, and the rest of her lab (BCM) for helpful discussion and for providing us with additional bacterial strains. We acknowledge the Baylor Molecular Bioscience Center (MBC) and the Center for Microscopy and Imaging (CMI) for providing instrumentation and resources aiding this project. We thank Oyindamola Adefisayo (Cornell Medicine) and all members of the Trakselis laboratory for productive conversations and insight. This work was funded by the NSF MCB (NSF1613534 to M.A.T.) and supported by Baylor University.

\section{References}

1. Trakselis MA. Structural mechanisms of hexameric helicase loading, assembly, and unwinding. F1000Research. 2016;5(111):1-12. doi: 10.12688/f1000research.7509.1. PubMed PMID: 26918187; PubMed Central PMCID: PMC4755419.

2. Patel SS, Donmez I. Mechanisms of helicases. J Biol Chem. 2006;281(27):18265-8. Epub 2006/05/04. doi: 10.1074/jbc.R600008200. PubMed PMID: 16670085.

3. Kaplan DL, Davey MJ, O'Donnell M. Mcm4,6,7 uses a "pump in ring" mechanism to unwind DNA by steric exclusion and actively translocate along a duplex. J Biol Chem. 2003;278(49):49171-82.

4. Carney SM, Gomathinayagam S, Leuba SH, Trakselis MA. Bacterial DnaB helicase interacts with the excluded strand to regulate unwinding. J Biol Chem. 2017;292(46):19001-12. Epub 2017/09/25. doi: 10.1074/jbc.M117.814178. PubMed PMID: 28939774; PubMed Central PMCID: PMCPMC5704481.

5. Graham BW, Schauer GD, Leuba SH, Trakselis MA. Steric exclusion and wrapping of the excluded DNA strand occurs along discrete external binding paths during MCM helicase unwinding. Nucleic Acids Res. 2011;39(15):6585-95. doi: 10.1093/nar/gkr345. PubMed PMID: 21576224; PubMed Central PMCID: PMCPMC3159478.

6. Graham BW, Tao Y, Dodge KL, Thaxton CT, Olaso D, Young NL, et al. DNA interactions probed by hydrogen-deuterium exchange (HDX) fourier transform ion cyclotron resonance mass spectrometry confirm external binding sites on the minichromosomal maintenance (MCM) helicase. J Biol Chem. 2016;291(24):12467-80. doi: 10.1074/jbc.M116.719591. PubMed PMID: 27044751; PubMed Central PMCID: PMCPMC4933441.

7. Rothenberg E, Trakselis MA, Bell SD, Ha T. MCM forked substrate specificity involves dynamic interaction with the 5'-tail. J Biol Chem. 2007;282(47):34229-34. doi: 10.1074/jbc.M706300200. PubMed PMID: 17884823.
8. Carney SM, Trakselis MA. The excluded DNA strand is SEW important for hexameric helicase unwinding. Methods. 2016;108:79-91. doi: 10.1016/j.ymeth.2016.04.008. PubMed PMID: 27068657.

9. McGeoch AT, Trakselis MA, Laskey RA, Bell SD. Organization of the archaeal MCM complex on DNA and implications for the helicase mechanism. Nat Struct Mol Biol. 2005;12(9):756-62. doi: 10.1038/nsmb974. PubMed PMID: 16116441.

10. Costa A, Ilves I, Tamberg N, Petojevic T, Nogales E, Botchan MR, et al. The structural basis for MCM2-7 helicase activation by GINS and Cde45. Nat Struct Mol Biol. 2011;18(4):471-7. doi: 10.1038/nsmb.2004. PubMed PMID: 21378962; PubMed Central PMCID: PMCPMC4184033.

11. Jeong YJ, Rajagopal V, Patel SS. Switching from single-stranded to doublestranded DNA limits the unwinding processivity of ring-shaped T7 DNA helicase. Nucleic Acids Res. 2013;41(7):4219-29. doi: 10.1093/nar/gkt133. PubMed PMID: 23446275; PubMed Central PMCID: PMCPMC3627605.

12. Lee SJ, Syed S, Enemark EJ, Schuck S, Stenlund A, Ha T, et al. Dynamic look at DNA unwinding by a replicative helicase. Proc Natl Acad Sci U S A. 2014;111(9):E827-35. doi: 10.1073/pnas.1322254111. PubMed PMID: 24550505; PubMed Central PMCID: PMC3948270.

13. Huang H, Zhao K, Arnett DR, Fanning E. A specific docking site for DNA polymerase \{alpha\}-primase on the SV40 helicase is required for viral primosome activity, but helicase activity is dispensable. J Biol Chem. 2010;285(43):33475-84. Epub 2010/08/06. doi: M110.156240 [pii]10.1074/jbc.M1 10.156240. PubMed PMID: 20685648; PubMed Central PMCID: PMC2963361.

14. Strycharska MS, Arias-Palomo E, Lyubimov AY, Erzberger JP, O'Shea VL, Bustamante CJ, et al. Nucleotide and partner-protein control of bacterial replicative helicase structure and function. Mol Cell. 2013;52(6):844-54. doi: 10.1016/j.molcel.2013.11.016. PubMed PMID: 24373746; PubMed Central PMCID: PMCPMC3929961.

15. Donmez I, Patel SS. Mechanisms of a ring shaped helicase. Nucleic Acids Res. 2006;34(15):4216-24. Epub 2006/08/29. doi: 10.1093/nar/gk1508. PubMed PMID: 16935879; PubMed Central PMCID: PMCPMC1616965.

16. Takahashi TS, Wigley DB, Walter JC. Pumps, paradoxes and ploughshares: Mechanism of the MCM2-7 DNA helicase. Trends Biochem Sci. 2005;30(8):437-44.

17. Singleton MR, Dillingham MS, Gaudier M, Kowalczykowski SC, Wigley DB. Crystal structure of RecBCD enzyme reveals a machine for processing DNA breaks. Nature. 2004;432(7014):187-93.

18. Li D, Zhao R, Lilyestrom W, Gai D, Zhang R, DeCaprio JA, et al. Structure of the replicative helicase of the oncoprotein SV40 large tumour antigen. Nature. 2003;423(6939):512-8.

19. Laskey RA, Madine MA. A rotary pumping model for helicase function of MCM proteins at a distance from replication forks. EMBO Rep. 2003;4(1):26-30.

20. Thomsen ND, Berger JM. Running in reverse: The structural basis for translocation polarity in hexameric helicases. Cell. 2009;139(3):523-34. Epub 2009/11/03. PubMed PMID: 19879839; PubMed Central PMCID: PMC2772833.

21. Lewis JS, Jergic S, Dixon NE. The E. coli DNA replication fork. Enzymes. 2016;39:31-88. Epub 2016/06/01. doi: 10.1016/bs.enz.2016.04.001. PubMed PMID: 27241927.

22. Mangiameli SM, Merrikh CN, Wiggins PA, Merrikh H. Transcription leads to pervasive replisome instability in bacteria. Elife. 2017;6:e19848. doi: 10.7554/eLife.19848. PubMed PMID: 28092263; PubMed Central PMCID: PMCPMC5305214.

23. Cox MM, Goodman MF, Kreuzer KN, Sherratt DJ, Sandler SJ, Marians KJ. The importance of repairing stalled replication forks. Nature. 2000;404(6773):37-41. Epub 2000/03/15. doi: 10.1038/35003501. PubMed PMID: 10716434.

24. Graham JE, Marians KJ, Kowalczykowski SC. Independent and stochastic action of DNA polymerases in the replisome. Cell. 2017;169(7):1201-13 e17. Epub 2017/06/18. doi: 10.1016/j.cell.2017.05.041. PubMed PMID: 28622507; PubMed Central PMCID: PMCPMC5548433.

25. Monachino E, Jergic S, Lewis JS, Xu ZQ, Lo ATY, O'Shea VL, et al. A primase-induced conformational switch controls the stability of the bacterial replisome. Mol Cell. 2020;79(1):140-+. doi: 10.1016/j.molcel.2020.04.037. PubMed PMID: WOS:000564562400005.

26. Arias-Palomo E, Puri N, O'Shea Murray VL, Yan Q, Berger JM. Physical basis for the loading of a bacterial replicative helicase onto DNA. Mol Cell. 
bioRxiv preprint doi: https://doi.org/10.1101/2021.05.27.445960; this version posted May 27, 2021. The copyright holder for this preprint (which was not certified by peer review) is the author/funder. All rights reserved. No reuse allowed without permission.

Behrmann et al., 25/05/2021 - preprint copy - BioRxiv

2019;74(1):173-84 e4. Epub 2019/02/25. doi: 10.1016/j.molcel.2019.01.023. PubMed PMID: 30797687; PubMed Central PMCID: PMCPMC6450724.

27. Kaplan DL, O'Donnell M. DnaB drives DNA branch migration and dislodges proteins while encircling two DNA strands. Mol Cell. 2002;10(3):647-57.

28. Byrne RT, Klingele AJ, Cabot EL, Schackwitz WS, Martin JA, Martin J, et al. Evolution of extreme resistance to ionizing radiation via genetic adaptation of DNA repair. Elife. 2014;3:e01322. Epub 2014/03/07. doi: 10.7554/eLife.01322. PubMed PMID: 24596148; PubMed Central PMCID: PMCPMC3939492.

29. Kim T, Chitteni-Pattu S, Cox BL, Wood EA, Sandler SJ, Cox MM. Directed evolution of RecA variants with enhanced capacity for conjugational recombination. PLoS genetics. 2015;11(6):e1005278. Epub 2015/06/06. doi: 10.1371/journal.pgen.1005278. PubMed PMID: 26047498; PubMed Central PMCID: PMCPMC4457935.

30. Travisano M. Long-term experimental evolution in Escherichia coli. VI. Environmental constraints on adaptation and divergence. Genetics. 1997;146(2):471-9. Epub 1997/06/01. PubMed PMID: 9177998; PubMed Central PMCID: PMCPMC1207989.

31. Bochner BR, Savageau MA. Generalized indicator plate for genetic, metabolic, and taxonomic studies with microorganisms. Appl Environ Microbiol. 1977;33(2):434-44. Epub 1977/02/01. doi:

10.1128/AEM.33.2.434-444.1977. PubMed PMID: 322611; PubMed Central PMCID: PMCPMC170700.

32. Tengerdy RP, Nagy JG, Martin B. Quantitative measurement of bacterial growth by the reduction of tetrazolium salts. Appl Microbiol. 1967;15(4):954-5. Epub 1967/07/01. PubMed PMID: 4860535; PubMed Central PMCID: PMCPMC547110.

33. Schleif R. Regulation of the L-arabinose operon of Escherichia coli. Trends Genet. 2000;16(12):559-65. Epub 2000/12/05. doi: 10.1016/s01689525(00)02153-3. PubMed PMID: 11102706.

34. Wiser MJ, Lenski RE. A comparison of methods to measure fitness in Escherichia coli. Plos One. 2015;10(5). doi: ARTN e0126210 10.1371/journal.pone.0126210. PubMed PMID: WOS:000354542500074.

35. Campbell EA, Korzheva N, Mustaev A, Murakami K, Nair S, Goldfarb A, et al. Structural mechanism for rifampicin inhibition of bacterial RNA polymerase. Cell. 2001;104(6):901-12. doi: Doi 10.1016/S00928674(01)00286-0. PubMed PMID: WOS:000167869200012.

36. Riber L, Lobner-Olesen A. Inhibition of Escherichia coli chromosome replication by rifampicin treatment or during the stringent response is overcome by de novo DnaA protein synthesis. Mol Microbiol. 2020;114(6):906-19. doi: 10.1111/mmi.14531. PubMed PMID: WOS:000540634300001.

37. Pierucci O. Chromosome replication and cell-division in Escherichia coli at various temperatures of growth. J Bacteriol. 1972;109(2):848-\&. doi: Doi 10.1128/Jb.109.2.848-854.1972. PubMed PMID: WOS:A1972L530700051.

38. Haugan MS, Charbon G, Frimodt-Moller N, Lobner-Olesen A. Chromosome replication as a measure of bacterial growth rate during Escherichia coli infection in the mouse peritonitis model. Sci Rep. 2018;8. doi: ARTN 14961 10.1038/s41598-018-33264-7. PubMed PMID: WOS:000446577500038.

39. Foster PL. Methods for determining spontaneous mutation rates. Methods Enzymol. 2006;409:195-213. Epub 2006/06/24. doi: 10.1016/S00766879(05)09012-9. PubMed PMID: 16793403; PubMed Central PMCID: PMCPMC2041832.

40. Wehrli W. Rifampin - Mechanisms of action and resistance. Rev Infect Dis. 1983;5:S407-S11. PubMed PMID: WOS:A1983RD00400003.

41. Simmons LA, Foti JJ, Cohen SE, Walker GC. The SOS regulatory network. EcoSal Plus. 2008;3(1). Epub 2008/09/01. doi: 10.1128/ecosalplus.5.4.3. PubMed PMID: 26443738.

42. Michel B. After 30 years of study, the bacterial SOS response still surprises us. PLoS Biol. 2005;3(7):e255. Epub 2005/07/08. doi: 10.1371/journal.pbio.0030255. PubMed PMID: 16000023; PubMed Central PMCID: PMCPMC1174825.

43. Chen SH, Byrne RT, Wood EA, Cox MM. Escherichia coli $\operatorname{radD}(y e j H)$ gene: a novel function involved in radiation resistance and double-strand break repair. Mol Microbiol. 2015;95(5):754-68. Epub 2014/11/27. doi: 10.1111/mmi.12885. PubMed PMID: 25425430; PubMed Central PMCID: PMCPMC4339530.
44. Verweij J, Pinedo HM. Mitomycin C: mechanism of action, usefulness and limitations. Anticancer Drugs. 1990;1(1):5-13. Epub 1990/10/01. PubMed PMID: 2131038.

45. Itsathitphaisarn O, Wing RA, Eliason WK, Wang J, Steitz TA. The hexameric helicase DnaB adopts a nonplanar conformation during translocation. Cell. 2012;151(2):267-77. doi: 10.1016/j.cell.2012.09.014. PubMed PMID: 23022319; PubMed Central PMCID: PMC3597440.

46. Perera HM, Behrmann MS, Hoang JM, Griffin WC, Trakselis MA. Contacts and context that regulate DNA helicase unwinding and replisome progression. Enzymes. 2019;45:183-223. Epub 2019/10/20. doi: 10.1016/bs.enz.2019.08.001. PubMed PMID: 31627877.

47. Tanner NA, Hamdan SM, Jergic S, Schaeffer PM, Dixon NE, van Oijen AM. Single-molecule studies of fork dynamics in Escherichia coli DNA replication. Nat Struct Mol Biol. 2008;15(2):170-6.

48. Kim S, Dallmann HG, McHenry CS, Marians KJ. Coupling of a replicative polymerase and helicase: A tau-DnaB interaction mediates rapid replication fork movement. Cell. 1996;84(4):643-50. Epub 1996/02/23. PubMed PMID: 8598050.

49. Yuan Q, Dohrmann PR, Sutton MD, McHenry CS. DNA polymerase III, but not polymerase IV, must be bound to a tau-containing DnaX complex to enable exchange into replication forks. J Biol Chem. 2016;291(22):1172735. doi: 10.1074/jbc.M116.725358. PubMed PMID: 27056333; PubMed Central PMCID: PMCPMC4882441.

50. Kath JE, Jergic S, Heltzel JM, Jacob DT, Dixon NE, Sutton MD, et al. Polymerase exchange on single DNA molecules reveals processivity clamp control of translesion synthesis. Proc Natl Acad Sci U S A. 2014;111(21):7647-52. doi: 10.1073/pnas.1321076111. PubMed PMID: 24825884; PubMed Central PMCID: PMC4040570.

51. Beattie TR, Kapadia N, Nicolas E, Uphoff S, Wollman AJ, Leake MC, et al. Frequent exchange of the DNA polymerase during bacterial chromosome replication. Elife. 2017;6:e21763. doi: 10.7554/eLife.21763. PubMed PMID: 28362256; PubMed Central PMCID: PMCPMC5403216.

52. Dewachter L, Verstraeten N, Fauvart M, Michiels J. An integrative view of cell cycle control in Escherichia coli. FEMS Microbiol Rev. 2018;42(2):116-36. Epub 2018/01/25. doi: 10.1093/femsre/fuy005. PubMed PMID: 29365084.

53. Lesterlin C, Gigant E, Boccard F, Espeli O. Sister chromatid interactions in bacteria revealed by a site-specific recombination assay. EMBO J. 2012;31(16):3468-79. Epub 2012/07/24. doi: 10.1038/emboj.2012.194. PubMed PMID: 22820946; PubMed Central PMCID: PMCPMC3419930.

54. Possoz C, Filipe SR, Grainge I, Sherratt DJ. Tracking of controlled Escherichia coli replication fork stalling and restart at repressor-bound DNA in vivo. EMBO J. 2006;25(11):2596-604. doi: 10.1038/sj.emboj.7601155. PubMed PMID: 16724111; PubMed Central PMCID: PMCPMC1478199.

55. Jameson KH, Wilkinson AJ. Control of initiation of DNA replication in Bacillus subtilis and Escherichia coli. Genes. 2017;8(1). doi: ARTN 22 10.3390/genes8010022. PubMed PMID: WOS:000399057100022.

56. Mulugu S, Potnis A, Shamsuzzaman, Taylor J, Alexander K, Bastia D. Mechanism of termination of DNA replication of Escherichia coli involves helicase-contrahelicase interaction. Proc Natl Acad Sci U S A. 2001;98(17):9569-74. doi: DOI 10.1073/pnas.171065898. PubMed PMID: WOS:000170539600022.

57. Kashav T, Nitharwal R, Abdulrehman SA, Gabdoulkhakov A, Saenger W, Dhar SK, et al. Three-dimensional structure of N-terminal domain of DnaB helicase and helicase-primase interactions in Helicobacter pylori. Plos One. 2009;4(10). doi: ARTN e7515 10.1371/journal.pone.0007515. PubMed PMID: WOS:000271012900013.

58. Thirlway J, Turner IJ, Gibson CT, Gardiner L, Brady K, Allen S, et al. DnaG interacts with a linker region that joins the $\mathrm{N}$ - and $\mathrm{C}$-domains of DnaB and induces the formation of 3 -fold symmetric rings. Nucleic Acids Res. 2004;32(10):2977-86. doi: 10.1093/nar/gkh628. PubMed PMID: WOS:000222118600009.

59. Lewis JS, Spenkelink LM, Jergic S, Wood EA, Monachino E, Horan NP, et al. Single-molecule visualization of fast polymerase turnover in the bacterial replisome. Elife. 2017;6:e23932. doi: 10.7554/eLife.23932. PubMed PMID: 28432790; PubMed Central PMCID: PMCPMC5419744.

60. Jiang W, Bikard D, Cox D, Zhang F, Marraffini LA. RNA-guided editing of bacterial genomes using CRISPR-Cas systems. Nat Biotechnol. 2013;31(3):233-9. Epub 2013/01/31. doi: 10.1038/nbt.2508. PubMed PMID: 23360965; PubMed Central PMCID: PMCPMC3748948. 
bioRxiv preprint doi: https://doi.org/10.1101/2021.05.27.445960; this version posted May 27, 2021. The copyright holder for this preprint (which was not certified by peer review) is the author/funder. All rights reserved. No reuse allowed without permission.

Behrmann et al., 25/05/2021 - preprint copy - BioRxiv

61. Costantino N, Court DL. Enhanced levels of lambda red-mediated recombinants in mismatch repair mutants. Proc Natl Acad Sci U S A. 2003;100(26):15748-53. doi: 10.1073/pnas.2434959100. PubMed PMID: WOS:000187554600083.

62. Lieber MR. The mechanism of double-strand DNA break repair by the nonhomologous DNA end-joining pathway. Annu Rev Biochem. 2010;79:181-211. doi: 10.1146/annurev.biochem.052308.093131. PubMed PMID: WOS:000280225300007.

63. Jasin M, Rothstein R. Repair of strand breaks by homologous recombination. Cold Spring Harbor perspectives in biology. 2013;5(11). doi: ARTN a012740 10.1101/cshperspect.a012740. PubMed PMID: WOS:000327742400004.

64. Bikard D, Jiang W, Samai P, Hochschild A, Zhang F, Marraffini LA. Programmable repression and activation of bacterial gene expression using an engineered CRISPR-Cas system. Nucleic Acids Res. 2013;41(15):742937. Epub 2013/06/14. doi: 10.1093/nar/gkt520. PubMed PMID: 23761437; PubMed Central PMCID: PMCPMC3753641.

65. Tjorve KMC, Tjorve E. The use of Gompertz models in growth analyses, and new Gompertz-model approach: An addition to the Unified-Richards family. Plos One. 2017;12(6). doi: ARTN e0178691

10.1371/journal.pone.0178691. PubMed PMID: WOS:000402837600030.
66. Rasband WS. ImageJ. Bethesda, Maryland, USA: U. S. National Institutes of Health; 1997-2016, 17 October 2015.

67. Carr KM, Kaguni JM. The A184V missense mutation of the dnaA5 and dnaA46 alleles confers a defect in ATP binding and thermolability in initiation of Escherichia coli DNA replication. Mol Microbiol. 1996;20(6):1307-18. doi: DOI 10.1111/j.1365-2958.1996.tb02649.x. PubMed PMID: WOS:A1996UU88000018.

68. Livak KJ, Schmittgen TD. Analysis of relative gene expression data using real-time quantitative PCR and the 2-[Delta][Delta]CT method. Methods. 2001;25(4):402-8. doi: DOI: 10.1006/meth.2001.1262.

69. Sutton MD, Duzen JM, Maul RW. Mutant forms of the Escherichia coli beta sliding clamp that distinguish between its roles in replication and DNA polymerase V-dependent translesion DNA synthesis. Mol Microbiol. 2005;55(6):1751-66. doi: 10.1111/j.1365-2958.2005.04500.x. PubMed PMID: 15752198.

70. Rohwer F, Azam F. Detection of DNA damage in prokaryotes by terminal deoxyribonucleotide transferase-mediated dUTP nick end labeling. Appl Environ Microbiol. 2000;66(3):1001-6. doi: Doi 10.1128/Aem.66.3.10011006.2000. PubMed PMID: WOS:000085604800020. 